\title{
Two-loop renormalization group restrictions on the standard model and the fourth chiral family
}

\author{
Yu. F. Pirogov \\ O. V. Zenin \\ Institute for High Energy Physics, \\ Protvino, Moscow Region, Russia \\ Moscow Institute of Physics and Technology, \\ Dolgoprudny, Moscow Region, Russia
}




\begin{abstract}
In the framework of the two-loop renormalization group, the global profile of the Standard Model (SM) in its full parameter space is investigated up to the scale of the gauge singularity. The critical Higgs masses bordering the strong coupling, unstable and safe regions are explicitly found. Restrictions on the Higgs boson mass as a function of a cutoff scale are obtained from the stability of electroweak vacuum and from the absence of the strong coupling both in the Higgs and Yukawa sectors. The cutoff being equal to the Planck scale requires the Higgs mass to be $M_{H}=(161.3 \pm 20.6)_{-10}^{+4} \mathrm{GeV}$ and $M_{H} \geq 140.7_{-10}^{+10}$ $\mathrm{GeV}$, where the $M_{H}$ corridor is the theoretical one and the errors are due to the top mass uncertainty.

The SM two-loop $\beta$ functions are generalized to the massive neutrino case. Modification of the two-loop global profile of the SM extended by one new chiral family is studied, and bounds on the masses of the family are derived. The requirement of self-consistency of the perturbative SM as an underlying theory up to the Planck or GUT scale excludes the fourth chiral family. But as an effective theory, the SM allows the heavy chiral family with the mass up to $250 \mathrm{GeV}$ depending on the Higgs mass and the cutoff scale. Under precision experiment restriction $M_{H} \leq 200 \mathrm{GeV}$, the fourth chiral family, taken alone, is excluded. Nevertheless a pair of the chiral families constituting the vector-like one could still exist.
\end{abstract}




\section{Introduction}

The renormalization group ( $\mathrm{RG}$ ) study of a field theory (for the review see, e.g., Refs. $[1,2])$ enables one to understand in grosso the structure of the theory as a function of a characteristic energy scale. Of special interest are the cases when self-consistency of the theory is under danger of violation. They may signal either the breakdown of the perturbative validity or/and the onset of a "new physics".

There are two problems of the kind in the Standard Model (SM). First, it encounters when some of the running couplings tend to blow up at the finite scales. The well-known examples are, e.g., the Landau singularity in QED (more generally, in any Abelian gauge theory $U(1)$ ) or in the $\phi^{4}$ scalar theory. In the latter case, the problem was known for a long time as the triviality problem (for the review of triviality arguments see, e.g., Ref. [3] and references therein). Technically, it can be hoped to be solved by an improvement of the perturbative series or by development of the strong coupling methods. But more probably it has a physical origin, and it could be solved eventually by the more complete theory which should result effectively in a physical cutoff (for an example see, e.g., Ref. [4]). In particular, this problem was invoked to justify the technicolor as a substitute for the heavy Higgs boson.

Second, the problem occurs when a running coupling leaves the physical region at some finite scale. In the SM, this happens when the Higgs quartic effective coupling becomes negative, indicating the absence of a ground state in the quantum theory. It is the so-called electroweak vacuum stability problem (for a review see, e.g., Ref. [5]). It is a real problem of the quantum field theory because this phenomenon takes place in the realm of the perturbative validity. In the framework of the SM, the light Higgs bosons resulting in the unstable electroweak vacuum should be forbidden. On the other hand, if this does happen some new scalar bosons beyond the SM will be required to stabilize the vacuum. Otherwise, the light composite Higgs with the compositeness scale corresponding to the scale of the stability breakdown might be a natural solution.

The SM self-consistency study in the framework of the one-loop RG and 
restrictions thereof on the SM heavy particles, the Higgs boson and the top quark, was undertaken in Refs. [3, 6, 7]. A generalization to the two-loop level was given in Refs. [8]-[10]. The one-loop RG restrictions on a new heavy chiral family were studied in Ref. [11], and that on the vector-like family were investigated in Ref. [12].

The aim of our present study is twofold. First, we investigate the two-loop RG global profile of the SM in its parameter space at all conceivable scales. In particular, we refine the $R G$ restrictions on the Higgs mass in the light of the now accurately known top mass and its uncertainty. This provides us with the background required for the RG study of possible extensions to the SM. Second, we generalize to the two-loop level the RG study of the SM extended by the fourth chiral family, as well as refine the self-consistency restrictions thereof on the Higgs and fourth family masses. This required in turn a generalization of the SM two-loop $\beta$ functions to the massive neutrino case, which we present. In a wider perspective, we consider the problem of what principally new the fourth heavy chiral family brings in the RG global profile of the SM.

\section{$2 \quad$ Standard Model}

The two-loop $\beta$ functions for a general gauge theory in the $\overline{\mathrm{MS}}$ renormalization scheme are well-known in the literature [13] as well as their particular realization for the SM [13]-[15] (compact summaries for the SM can also be found in Refs. [16, 17]). They are re-collected in a different form in the Appendix A1 with the explicit Yukawa couplings being retained only for the third family. In what follows we put just the generic structure of the emerging one- and two-loop RG differential equations.

Let $g_{i}, i=1,2,3, y_{f}, \lambda$ and $v$ be the SM gauge couplings, the Yukawa couplings for fermions $f$, the Higgs self-interaction coupling and the vacuum expectation value $(\mathrm{VEV})$, respectively. Then one gets

$$
\begin{aligned}
\frac{1}{g_{i}^{3}} \frac{d g_{i}}{d \ln \mu} & =\frac{1}{(4 \pi)^{2}} b_{g_{i}}^{(1)}\left(g_{i^{\prime}}\right)+\frac{1}{(4 \pi)^{4}} b_{g_{i}}^{(2)}\left(g_{i^{\prime}}, y_{f^{\prime}}\right), \\
\frac{1}{y_{f}} \frac{d y_{f}}{d \ln \mu} & =\frac{1}{(4 \pi)^{2}} b_{y_{f}}^{(1)}\left(g_{i^{\prime}}, y_{f^{\prime}}\right)+\frac{1}{(4 \pi)^{4}} b_{y_{f}}^{(2)}\left(g_{i^{\prime}}, y_{f^{\prime}}, \lambda\right),
\end{aligned}
$$




$$
\begin{aligned}
\frac{d \lambda}{d \ln \mu} & =\frac{1}{(4 \pi)^{2}} b_{\lambda}^{(1)}\left(g_{i^{\prime}}, y_{f^{\prime}}, \lambda\right)+\frac{1}{(4 \pi)^{4}} b_{\lambda}^{(2)}\left(g_{i^{\prime}}, y_{f^{\prime}}, \lambda\right), \\
\frac{1}{v} \frac{d v}{d \ln \mu} & =\frac{1}{(4 \pi)^{2}} b_{v}^{(1)}\left(g_{i^{\prime}}, y_{f^{\prime}}\right)+\frac{1}{(4 \pi)^{4}} b_{v}^{(2)}\left(g_{i^{\prime}}, y_{f^{\prime}}, \lambda\right),
\end{aligned}
$$

where $\mu$ is a renormalization scale, say in $\mathrm{GeV}, b^{(1)}$ and $b^{(2)}$ are one- and two-loop contributions respectively, $b_{g_{i}}^{(1)}$ being in fact the constants. Under $g_{i^{\prime}}$ and $y_{f^{\prime}}$ there are understood the sets of all $g_{i}$ and $y_{f}$. We neglected here for simplicity by the mixing of the Yukawa couplings and thus by the $\mathrm{CP}$ violating phase. To state it in other terms, the diagonal real form of the Yukawa matrix $Y_{f f^{\prime}}=y_{f} \delta_{f f^{\prime}}$ is implied.

The following essential features of the RG system (1) are readily ascertained. At one-loop order, one has a kind of the three-level up-down hierarchy among the SM couplings, so that the first three differential equations in (1) disentangle. One can first find $g_{i}(\mu)$, then insert them into $\beta_{y_{f}}^{(1)}$ and find $y_{f}(\mu)$, and finally put $g_{i}(\mu), y_{f}(\mu)$ into the third equation and integrate it. The solution to the equation for $v(\mu)$ is determined completely by those for the first three equations, both in one and two loops. ${ }^{1}$

In two loops, the RG equations partially entangle with each other due to a down-up feedback to the neighbour level: from $\lambda$ to $y_{f}$ and from $y_{f}$ to $g_{i}$. But there is no direct influence of $\lambda$ on $g_{i}$. It emerges only in three loops. Hence to completely entangle the RG system one needs the three-loop SM $\beta$ functions, which are unknown at present. Thus we have to restrict ourselves to the twoloop order. On the other hand, the two- and higher-loop contributions to $\beta$ functions, even the sign including, are known to depend in a multi-coupling theory on the renormalization scheme [2]. Hence the physical meaning of the running couplings becomes ambiguous, and it is impossible to improve the perturbative RG analysis of the SM in the scheme-independent way beyond one loop.

We integrated the RG Eq. (1) numerically for $\mu \geq M_{Z}$ by the first-order Runge-Cutta method with the initial conditions at the scale $M_{Z}$ taken as

$$
\begin{aligned}
& \alpha_{1}\left(M_{Z}\right)=0.0102, \\
& \alpha_{2}\left(M_{Z}\right)=0.0338,
\end{aligned}
$$

\footnotetext{
${ }^{1}$ For the sake of completeness, the evolution of the gauge fixing parameter $\xi$ should also be taken into account in the gauge dependent quantities like $v(\mu)$. To avoid this one can use the 't Hooft-Landau gauge $\xi=0$ which is not running.
} 


$$
\alpha_{3}\left(M_{Z}\right)=0.123
$$

in accordance with $\alpha\left(M_{Z}\right)=1 / 127.90$ and $\sin ^{2} \theta_{W}\left(M_{Z}\right)=0.2315$ [18]. Our normalizations of the gauge couplings are as follows: $g_{1}=(5 / 3)^{1 / 2} g^{\prime}, g_{2} \equiv g$ and $g_{3} \equiv g_{S}$, with $g^{\prime}, g$ and $g_{S}$ being the conventional SM couplings. We choose also the relations $m_{f}=y_{f} v$ and $m_{H}=\lambda^{1 / 2} v$ as the definition of normalization for the Higgs and Yukawa couplings, with $v=\left(\sqrt{2} G_{F}\right)^{-1 / 2}=$ $246.22 \mathrm{GeV}$ being the Higgs VEV. Because the evolution of $v(\mu)$ is gauge dependent we use in what follows only the gauge independent observable $v \equiv v\left(M_{Z}\right)$.

Besides, we use at $\mu=M_{Z}$ the one-loop matching condition for the physical $M_{f}$ and running $m_{f}(\mu) \equiv y_{f}(\mu) v$ masses of the fermions $f=q$ and $l$ given by

$$
m_{f}(\mu)=M_{f}\left(1+\delta_{f}^{\mathrm{QCD}}(\mu)+\delta_{f}^{\mathrm{QED}}(\mu)+\delta_{f}^{(\mathrm{t}, \mathrm{H})}(\mu)\right) .
$$

Here one has

$$
\delta_{q}^{\mathrm{QCD}}(\mu)=-\frac{4}{3} \frac{\alpha_{3}(\mu)}{\pi}\left(1+\frac{3}{4} \ln \frac{\mu^{2}}{M_{q}^{2}}\right),
$$

with $\delta_{f}^{\mathrm{QED}}$ obtained from the last Eq. by substituting $4 / 3 \alpha_{3}$ by $Q_{f}^{2} \alpha$, where $Q_{f}$ is the electric charge of the fermion $f$. The top quark and Higgs boson induced radiative corrections $\delta_{f}^{(\mathrm{t}, \mathrm{H})}$ can be found in Ref. [19] as

$$
\begin{aligned}
\delta_{\tau}^{(\mathrm{t}, \mathrm{H})}(\mu) & =\frac{1}{(4 \pi)^{2}}\left(\frac{M_{t}}{v}\right)^{2}\left(3 \ln \frac{\mu^{2}}{M_{t}^{2}}+\frac{3}{2}+\frac{1}{4} \frac{M_{H}^{2}}{M_{t}^{2}}\right), \\
\delta_{b}^{(\mathrm{t}, \mathrm{H})}(\mu) & =\frac{1}{(4 \pi)^{2}}\left(\frac{M_{t}}{v}\right)^{2}\left(\frac{3}{2} \ln \frac{\mu^{2}}{M_{t}^{2}}+\frac{1}{4}+\frac{1}{4} \frac{M_{H}^{2}}{M_{t}^{2}}\right), \\
\delta_{t}^{(\mathrm{t}, \mathrm{H})}(\mu) & =\frac{1}{(4 \pi)^{2}}\left(\frac{M_{t}}{v}\right)^{2}\left(\frac{9}{2} \ln \frac{\mu^{2}}{M_{t}^{2}}+\frac{11}{2}-2 \pi \frac{M_{H}}{M_{t}}\right),
\end{aligned}
$$

where the last line is valid at $\left(M_{H} / 2 M_{t}\right)^{2} \ll 1$. Similarly, the initial value $m_{H}\left(M_{Z}\right)$ is related with the physical Higgs mass $M_{H}$ through the running mass $m_{H}(\mu) \equiv y_{H}(\mu) v$ at the scale $M_{Z}$, where

$$
m_{H}(\mu)=M_{H}\left(1+\delta_{H}(\mu)\right) \text {. }
$$


In the limit $\left(M_{H} / 2 M_{t}\right)^{2} \ll 1$ one has the following asymptotic one-loop expression $[19,20]$

$$
\begin{aligned}
(4 \pi)^{2} \delta_{H}(\mu)= & \left(\frac{M_{H}}{v}\right)^{2}\left(3 \ln \frac{\mu^{2}}{M_{H}^{2}}+6-\frac{3 \sqrt{3} \pi}{4}\right) \\
+ & \left(\frac{M_{t}}{v}\right)^{2}\left(3 \ln \frac{\mu^{2}}{M_{t}^{2}}-2+\frac{3}{10} \frac{M_{H}^{2}}{M_{t}^{2}}\right) \\
+ & \left(\frac{M_{W}}{v}\right)^{2}\left[3 \ln \frac{M_{W}^{2}}{\mu^{2}}+\frac{1}{2} \frac{M_{H}^{2}}{M_{W}^{2}} \ln \frac{M_{H}^{2}}{M_{W}^{2}}-5+12 \frac{M_{W}^{2}}{M_{H}^{2}}\right. \\
& \left.\quad-4\left(3 \frac{M_{W}^{2}}{M_{H}^{2}}+\frac{1}{4} \frac{M_{H}^{2}}{M_{W}^{2}}-1\right) f\left(\frac{M_{W}^{2}}{M_{H}^{2}}\right)\right] \\
+ & \left(\frac{M_{Z}}{v}\right)^{2}\left[\frac{3}{2} \ln \frac{M_{Z}^{2}}{\mu^{2}}+\frac{1}{4} \frac{m_{H}^{2}}{M_{Z}^{2}} \ln \frac{M_{H}^{2}}{M_{Z}^{2}}-\frac{5}{2}+6 \frac{M_{Z}^{2}}{M_{H}^{2}}\right. \\
& \left.-2\left(3 \frac{M_{Z}^{2}}{M_{H}^{2}}+\frac{1}{4} \frac{M_{H}^{2}}{M_{Z}^{2}}-1\right) f\left(\frac{M_{Z}^{2}}{M_{H}^{2}}\right)\right]
\end{aligned}
$$

where

$$
f(x)= \begin{cases}(4 x-1)^{1 / 2} \operatorname{arctg}(4 x-1)^{-1 / 2}, & x>\frac{1}{4} \\ \frac{1}{2}(1-4 x)^{1 / 2} \ln \frac{1+(1-4 x)^{1 / 2}}{1-(1-4 x)^{1 / 2}}, & x<\frac{1}{4} .\end{cases}
$$

Putting all together one gets finally at $M_{H}=150 \mathrm{GeV}$ :

$$
\begin{aligned}
& m_{\tau}\left(M_{Z}\right)=1.764 \mathrm{GeV}, \\
& m_{b}\left(M_{Z}\right)=(4.47 \pm 0.50) \mathrm{GeV}, \\
& m_{t}\left(M_{Z}\right)=\left(171.8_{-4.7}^{+4.6}\right) \mathrm{GeV} .
\end{aligned}
$$

The last two values correspond in turn to the physical bottom and top masses $M_{b}=(4.5 \pm 0.5) \mathrm{GeV}[18]$ and $M_{t}=(175 \pm 5) \mathrm{GeV}$ [21], respectively. Only errors in the top mass are left as the main source of the subsequent uncertainties.

As a field theory, SM is legitimate to be pulled to its inner ultimate limits. This may help to understand better its structure in the physically reasonable region $\mu<M_{P l}, M_{P l} \simeq 10^{19} \mathrm{GeV}$, which is to be considered more seriously. So all the subsequent numerical results are obtained at all allowed $\mu$ with the exact two-loop $\beta$ functions. Most of the terms in the latter ones proved to be 
crucial for the quantitative evolution of couplings in the physical $\mu$ region up to the Planck scale. But for the qualitative analysis of the SM RG solutions at extremely high $\mu, \mu \gg M_{P l}$, we retain in the coefficients of the $\beta$ functions given below only the most representative terms.

To estimate the dependence of the results on the loop order and to pick out regions where perturbation theory may be more reliably trusted, we present both the one- and two-loop results. They are shown in Figs. 1-5. Let us discuss them in turn for the gauge, Yukawa and Higgs sectors of the SM.

\section{(i) Gauge sector}

Fig. 1 shows the running with $\mu$ of the inverse gauge couplings squared. Under simplifications adopted, one has (with the number of generations here and in what follows $n_{g}=3$ )

$$
\begin{aligned}
(4 \pi)^{2} \beta_{g_{1}}^{(1)} & =\frac{41}{10} \\
(4 \pi)^{4} \beta_{g_{1}}^{(2)} & =\frac{199}{50} g_{1}^{2}-\frac{17}{5} y_{t}^{2}+\cdots
\end{aligned}
$$

It can be seen that at the one-loop order the coupling $g_{1}$ develops a pole singularity at $\Lambda_{g_{1}}^{(1)}, \log \Lambda_{g_{1}}^{(1)}=41$. Validity of the perturbation theory in $g_{1}$ restricts $\alpha_{1} \leq 4 \pi$ and hence $\log \mu \leq 40$, which is in the logarithmic scale twice as large as the Planck scale. We should assume this restriction on the physical grounds in what follows. Nevertheless, taken at its face value the two-loop RG has the meaning by itself. So in order to understand the mathematical structure of its solutions better, we extend them up to the singularity point $\Lambda_{g_{1}}$.

As it is seen from Fig. 1, the actual influence of $y_{f}(\lambda(\mu))$ on $g_{1}$ in two loops is somewhat sizable only for heavy Higgs. It diminishes the slope of $g_{1}(\mu)$ at $\mu$ beyond the Planck scale, where $y_{f}$ are large, and shifts the singularity position $\Lambda_{g_{1}}^{(2)}$ upwards to $\log \Lambda_{g_{1}}^{(2)}=47$ for the heavy Higgs $\left(m_{H}\left(M_{Z}\right)=450 \mathrm{GeV}\right)$, which is close to that maximally allowed by the perturbative consistency in $\lambda$. The value $m_{H}\left(M_{Z}\right)=136.1 \mathrm{GeV}$ corresponds to the highest lower bound of the electroweak vacuum stability (see later on). Curves corresponding to the lighter Higgs bosons are very close to that for $m_{H}\left(M_{Z}\right)=136.1 \mathrm{GeV}$. Curves for the intermediate values of $m_{H}\left(M_{Z}\right)$, i.e. $136.1 \mathrm{GeV}<m_{H}\left(M_{Z}\right)<450$ 
$\mathrm{GeV}$, are located in between the two extreme cases. In other respects the picture in Fig. 1 is well-known.

\section{(ii) Yukawa sector}

Fig. 2 depicts the evolution of the Yukawa couplings $y_{f}$ for the third family SM fermions: $t, b$ quarks, and $\tau$ lepton, with the fact of the $t$ quark being heavy taken into account. One has approximately for the top quark

$$
\begin{aligned}
& (4 \pi)^{2} \beta_{y_{t}}^{(1)}=9 y_{t}^{2}-\frac{17}{20} g_{1}^{2}-\frac{9}{4} g_{2}^{2}-8 g_{3}^{2}+\cdots \\
& (4 \pi)^{4} \beta_{y_{t}}^{(2)}=-48 y_{t}^{4}+\frac{3}{2} \lambda^{2}-12 y_{t}^{2} \lambda+\cdots .
\end{aligned}
$$

In one loop, $\beta_{f}^{(1)}$ are dominated by the negative gauge contributions, so that all the $y_{f}$ are falling down with $\mu$ and lie in the weak coupling regime. ${ }^{2}$

But in two loops the behaviour changes drastically. An approximate UV stable fixed point appears at $y_{t}^{(\mathrm{UV})} \simeq 5.4$ due to compensation of the $y_{t}^{2}$ and $y_{t}^{4}$ contributions. In the real world, this critical value is approached from below both for $t, b$ quarks and for $\tau$ lepton, the faster the heavier Higgs boson is. Hence for the sufficiently heavy Higgs, $m_{H}\left(M_{Z}\right) \geq 200 \mathrm{GeV}$, all the third family fermions would fall into the strong coupling regime at sufficiently high $\mu$. This would make the third family fermions much more alike at the high scales than at the electroweak one. In practice, prior to $M_{P l}$ the strong coupling develops only for $t$ quark when Higgs is rather heavy, $m_{H}\left(M_{Z}\right) \geq 450 \mathrm{GeV}$. Because from the combined LEP data on the precision experiments it follows that $M_{H} \leq 200 \mathrm{GeV}$ at $95 \%$ C.L. [22], one may conclude that the Yukawa sector of the SM is weakly coupled along all the physically reasonable region of $\mu, \mu \leq M_{P l}$.

\footnotetext{
${ }^{2}$ To be more precise, the one-loop trajectory for the $\tau$ lepton is mildly convex, so that it intersects with the curve for the $b$ quark near the GUT scale.
} 


\section{(iii) Higgs sector}

Fig. 3 presents running of the Higgs quartic coupling. One has approximately for it

$$
\begin{aligned}
& (4 \pi)^{2} \beta_{\lambda}^{(1)}=12 \lambda^{2}-48 y_{t}^{4}+\frac{27}{100} g_{1}^{4}+\left(24 y_{t}^{2}-\frac{9}{5} g_{1}^{2}\right) \lambda+\cdots \\
& (4 \pi)^{4} \beta_{\lambda}^{(2)}=-78 \lambda^{3}-3.411 g_{1}^{6}+\cdots
\end{aligned}
$$

The $\beta$ function for the pure Higgs sector is known in the $\overline{\mathrm{MS}}$ scheme up to the three-loop order [23]

$$
(4 \pi)^{6} \beta_{\lambda}^{(3)}=(897+504 \zeta(3)) \lambda^{4}
$$

being scheme dependent. Fig. 3a shows the one-loop behaviour of $\lambda^{1 / 2}$. It is seen that for $m_{H}>m_{H \text { min }}^{(1)}=142.7 \mathrm{GeV}$ a singularity in $\lambda$ develops at $\log \Lambda_{\lambda}^{(1)} \leq 41$. On the other hand, the theory at $m_{H}\left(M_{Z}\right)<m_{H \min }^{(1)}$ possesses the unstable Higgs vacuum with $\lambda<0$ due to the negative top quark contribution $\sim y_{t}^{4}$.

In two loops, there are three critical curves shown in bold in Fig. 3b. First of all, there appears an approximate UV stable fixed point at $\lambda_{\mathrm{UV}}^{1 / 2} \simeq 4.93$ produced by the compensation of the one- and two-loop terms: $\lambda^{2}$ and $\lambda^{3}$. It corresponds to boundary value of the Higgs mass $m_{H}^{(2)}{ }_{\max }\left(M_{Z}\right)=1200 \mathrm{GeV}$, at and above which the theory is definitely strongly coupled. The boundary Higgs mass for the vacuum instability shifts in two loops to $m_{H \text { min }}^{(2)}\left(M_{Z}\right)=$ $136.1 \mathrm{GeV}$. The third critical value $m_{H}^{(2)}{ }_{\text {inter }}\left(M_{Z}\right)=156.7 \mathrm{GeV}$ borders the region with the potentially strongly coupled Higgs from the one with the weakly coupled Higgs. Note that theory with $m_{H \text { min }}^{(2)}<m_{H}\left(M_{Z}\right)<m_{H}^{(2)}$ inter is consistent in two loops up to the ultimate scale $\mu=\Lambda_{g_{1}}^{(2)}$.

Finally, one can impose the requirement of the SM self-consistency up to some cutoff scale $\Lambda$. In other terms, the theory should be neither strongly coupled nor unstable at $\mu \leq \Lambda$. In one loop, this means that the $\lambda$ singularity position fulfills the requirement $\Lambda_{\lambda}^{(1)} \geq \Lambda$, and simultaneously one has $\left.\mu\right|_{\lambda=0} \geq \Lambda$. In two loops, we should choose as a criterion for the onset of the strong coupling regime the requirements $\beta_{\lambda}^{(2)} /\left.\beta_{\lambda}^{(1)}\right|_{\Lambda}$ and $\beta_{\lambda}^{(3)} /\left.\beta_{\lambda}^{(2)}\right|_{\Lambda}<1$ which could guarantee the perturbativity and the scheme independence. In neglect by all the couplings but $\lambda$ this would mean that $\lambda^{1 / 2} \leq 2$, in particular 
$m_{H}\left(M_{Z}\right) \leq 500 \mathrm{GeV}$, the restriction we retain for the whole SM. Not knowing $\beta_{t}^{(3)}$ we restrict ourselves just by the requirement that $\beta_{t}^{(2)} /\left.\beta_{t}^{(1)}\right|_{\Lambda} \ll 1$ which is definitely fulfilled at $y_{t} \leq 2<y_{t}^{(U V)}$.

The one- and two-loop restrictions are drawn in Fig. 4. Here use is made of the exact one-loop Eq. (6) for transition from the $\overline{\mathrm{MS}}$ value $m_{H}\left(M_{Z}\right)$ to the physical Higgs mass $M_{H}$. The sensitivity of the allowed region of the Higgs mass to the uncertainty of the top quark mass is also indicated. Strictly speaking, allowed is the region between the most upper and the most lower curves. This means that for $\Lambda=M_{P l}$ the legitimate Higgs mass is $M_{H}=(161.3 \pm 20.6)_{-10}^{+4} \mathrm{GeV}$. One gets also the lower bound $M_{H} \geq 140.7 \pm 10$ $\mathrm{GeV}$ at such a cutoff. The allowed Higgs mass region is much wider for a lower cutoff scale $\Lambda .^{3}$ For completeness, we present in Fig. 5 the plot for $v(\mu)$ both in one and two loops. It is seen that the electroweak symmetry never restores prior to the Plank scale.

\section{The fourth chiral family}

The two-loop RG global profile of the SM being understood, one is in a position to discuss the SM conceivable extensions. We consider here the minimum SM extension by means of the additional heavy fermion families. If alone, the fourth family should have with necessity the same chirality pattern as the three light families. This is to be required to avoid the potential problem of the large direct mass mixing for the fourth family with the light ones.

What concerns the fifth family, there are two possibilities: either it has the same chirality as the four previous families, or it is a mirror one (or to state it differently, it is charge conjugate with respect to the rest of the families). In the first case, the analysis repeats itself just with more parameters. In the second case, the large direct mass terms could be introduced for the pair of the fourth and fifth families, in addition to Yukawa couplings. This

\footnotetext{
${ }^{3}$ The true condition for the electroweak vacuum stability is the existence of a global minimum in the Higgs effective potential [24, 25]. For the two-loop RG improved one-loop effective potential $V_{e f f}(\mu, \phi)$ this turns out at $\Lambda \simeq M_{P l}$ to be in practice equivalent to our requirement that the running coupling does not become negative. At the lower values of $\Lambda$ there are discrepancies which we attribute to the difference of the stability criteria.
} 
proliferates enormously the number of free parameters and makes the general analysis impossible. On the other hand, if one chooses a mass independent renormalization scheme, say $\overline{\mathrm{MS}}$, the net influence of the direct mass terms on the evolution of the SM parameters will be just in the threshold effects. Barring them, this case, which may likewise be attributed to one vector-like family, is technically equivalent to the case with two chiral families.

For these reasons, we restrict ourselves by one new chiral family. In order to conform with experimental value for the number of light neutrinos $\left(n_{\nu}=3\right)$, we should add also the right-handed neutrinos $\nu_{R}$ (at least for the fourth family) and the proper Yukawa couplings for them. The right-handed neutrinos may possess the explicit Majorana mass as well, so that the physical neutrino masses may be quite different from their Yukawa counterparts. Because in the mass independent renormalization the explicit mass terms are important only in the threshold effects, we disregard them in what follows. We generalized the two-loop SM $\beta$ functions of Ref. [13] to the case with the neutrino Yukawa couplings. The results are given in the Appendix A2. The rest of the $\beta$ functions is as in Ref. [13]. For practical calculations with the fourth family we neglected by the light neutrino Yukawa couplings. The reduced four family $\beta$ functions are given in Appendix A3.

At present, there are no theoretical hints on the existence (or v.v.) of the fourth (and the higher) family. Nevertheless, one can extract some restrictions on the corresponding fermion masses. They are twofold, the direct and indirect ones, being in a sense complementary to each other. The first group gives bounds on the common mass scale of the fourth family, the second one restricts the splitting of the masses inside the family.

The existing direct experimental bounds on the masses of the fourth family quarks $t_{4}$ and $b_{4}$ depend somewhat on the assumptions about their decays. If the lightest of the quarks, say $b_{4}$, is stable enough to leave the detector, the limit on its mass is $M_{4} \geq 140 \mathrm{GeV}$ [26]. On the other hand, for unstable quarks, decaying inside the detector, the limit can be estimated from the CDF and D0 searches for the top quark [21] to be about $M_{t}$. What concerns the neutral and charged leptons of the fourth family, $\nu_{4}$ and $e_{4}$, it follows from LEP searches that $M_{\nu_{4}} \geq 59 \mathrm{GeV}$ and $M_{e_{4}} \geq 90 \mathrm{GeV}$ at $95 \%$ C.L. [18, 27].

The indirect restrictions can be extracted from the precision electroweak data, and they are related to the absence of decoupling with respect to the heavy chiral fermions. This results in the quadratically growing dependence of the electroweak radiative corrections on the heavy fermion masses. To 
avoid such a large corrections, as the precision data require, the members of a heavy fermion doublet should be highly degenerate. Namely, one should have for the quarks $t_{4}$ and $b_{4}$ that $\left(M_{t_{4}}^{2}-M_{b_{4}}^{2}\right) / M_{Z}^{2} \leq 1$, and similarly for the leptons $\nu_{4}, e_{4} \cdot{ }^{4}$

To reduce the number of free parameters we assume in what follows that $m_{t_{4}}=m_{b_{4}}=m_{Q}$ and $m_{\nu_{4}}=m_{e_{4}}=m_{L}$. As representative, we considered two cases: $m_{L} / m_{Q}=1 / 2$ and 1 , with the common mass $m_{Q}$ of the heavy quarks given by the fourth family scale $m_{4}$. It follows that both these typical cases do not contradict to the direct experimental bounds if $m_{4} \geq 180 \mathrm{GeV}$. Our results for the case $m_{4}=200 \mathrm{GeV}$, which we consider as more realistic, are presented in Figs. 6-9. Cases $a$ correspond to $m_{L} / m_{Q}=1 / 2$ and cases $b$ to $m_{L} / m_{Q}=1$.

Fig. 6 shows the evolution of $\alpha_{i}^{-1}$ with $\mu .^{5}$ It is seen that the two-loop contributions manifest themselves at rather low scales, $\mu=\left(10^{7}-10^{8}\right) \mathrm{GeV}$. They are governed by the onset of the strong coupling regime in the Yukawa sector at such a $\mu$ (see Fig. 7). Accordingly, the perturbatively consistent region of $\mu$ in the Higgs sector shrinks to the same values (see Fig. 8). Applying now the same criteria of self-consistency as in the case of the minimal SM we get the allowed values of $M_{H}$ depending on the cutoff scale $\Lambda$ (Fig. 9). The sensitivity to the shift in the mass $m_{4}$ is also indicated. The dependence on $\Delta M_{t}$ is much smaller, and it is not shown.

Finally, Figs. 10-11 present the one- and two-loop allowed regions in the $m_{4}-M_{H}$ plane. The influence of the Yukawa perturbative validity in two loops on the allowed regions of $m_{4}$ and $M_{H}$ is rather weak at the high $\Lambda$. Fig. 11 excludes the fourth heavy chiral family at high $\Lambda, \Lambda \geq 10^{10} \mathrm{GeV}$, independent of the Higgs mass. Under LEP restriction $M_{H} \leq 200 \mathrm{GeV}$, the fourth chiral family is completely excluded. ${ }^{6}$ Dependence of the restrictions on the top nery faint.

${ }^{4}$ One important peculiarity of the vector-like family is the decoupling with respect to the explicit mass term, at the Yukawa couplings being fixed. Hence, unlike the chiral family case, there is no need here for the high degeneracy in the Yukawa couplings to suppress the large radiative corrections.

${ }^{5}$ It may be noted that the GUT triangle shrinks but the conceivable gauge unification takes place beyond the region of perturbativity both in Yukawa and Higgs sectors.

${ }^{6}$ Though one possible caveat emerges if one adopts that the fourth family is vector-like and that, unnatural as it may seem, its Yukawa couplings are small. Then the ensuing restrictions on the family are strongly reduced, and the vector-like fourth family could exist. 


\section{Conclusions}

Let us summarize the differences in the RG global profiles of the SM with three and four chiral generations. For three generations with the experimentally known masses, the Yukawa sector is weakly coupled in the one-loop approximation. Prior to the Planck scale, the strong coupling may appear in one loop only in the Higgs self-interactions for the sufficiently heavy Higgs. It drives strong coupling for the Yukawa sector as well, but only through two loops. As a result, this influence is reduced, and the Yukawa sector stays weakly coupled up to the Planck scale for all experimentally preferred values of the Higgs mass, $M_{H} \leq 200 \mathrm{GeV}$. Validity of the perturbative SM up to the Planck scale, the Yukawa sector including, as well as the vacuum stability require the Higgs mass to be $M_{H}=(161.3 \pm 20.6)_{-10}^{+4} \mathrm{GeV}$ and $M_{H} \geq 140.7_{-10}^{+10}$ $\mathrm{GeV}$. Here the $M_{H}$ corridor is the theoretical one with the errors being produced by the top mass uncertainty. The allowed Higgs mass region is wider for a lower cutoff scale $\Lambda$.

The inclusion of the fourth heavy chiral family qualitatively changes the mode of the SM realization. With the addition of the family, the strong coupling is driven in one loop by the Yukawa interactions. It transmits to the Higgs self-interactions at the one-loop order, too. Hence the strong coupling develops in both these sectors in parallel, and their couplings blow up at

sufficiently low scales. As a result, the requirement of self-consistency of the perturbative SM as an underlying theory up to the Planck or GUT scale excludes the fourth chiral family. But as an effective theory, the SM allows the heavy chiral family with the mass up to $250 \mathrm{GeV}$ depending on the Higgs mass and the cutoff scale. Under precision experiment restriction $M_{H} \leq 200 \mathrm{GeV}$, the fourth chiral family, taken alone, is excluded. Nevertheless a pair of the chiral families constituting the vector-like one could still exist.

Acknowledgement This work was supported by the RFBR under grant No. $96-02-18122$.

Note added in the 2nd version of the e-print The authors wish to thank Akin Wingerter for the discussion resulted in a correction of misprints in contributions to $\beta_{\mathbf{Y}_{e}}^{(2)}$ induced by heavy neutrino (pp. 16, 18). 


\section{Appendices}

\section{A1 SM $\beta$ functions}

Here $n_{g}=3$ and the generation index $g$ runs over the values $g=1,2,3$. One has in fact $\sum_{g} \equiv \delta_{g 3}$. Here and in what follows the parts of the expressions for the anomalous dimension $\gamma_{v}$ which are proportional to the gauge couplings are valid in the 't Hooft-Landau gauge $\xi=0$.

\section{One-loop contributions}

(i) Gauge sector:

$$
\begin{aligned}
& (4 \pi)^{2} g_{1}^{-3} \beta_{g_{1}}^{(1)}=\frac{41}{10}, \\
& (4 \pi)^{2} g_{2}^{-3} \beta_{g_{2}}^{(1)}=-\frac{19}{6}, \\
& (4 \pi)^{2} g_{3}^{-3} \beta_{g_{3}}^{(1)}=-7 .
\end{aligned}
$$

(ii) Yukawa sector:

$$
\begin{aligned}
& (4 \pi)^{2} y_{\tau}^{-1} \beta_{y_{\tau}}^{(1)}=3 y_{\tau}^{2}+2 \sum_{g}\left(3 y_{u_{g}}^{2}+3 y_{d_{g}}^{2}+y_{e_{g}}^{2}\right)-\frac{9}{4} g_{1}^{2}-\frac{9}{4} g_{2}^{2}, \\
& (4 \pi)^{2} y_{t}^{-1} \beta_{y_{t}}^{(1)}=3 y_{t}^{2}-3 y_{b}^{2}+2 \sum_{g}\left(3 y_{u_{g}}^{2}+3 y_{d_{g}}^{2}+y_{e_{g}}^{2}\right)-\frac{17}{20} g_{1}^{2}-\frac{9}{4} g_{2}^{2}-8 g_{3}^{2}, \\
& (4 \pi)^{2} y_{b}^{-1} \beta_{y_{b}}^{(1)}=3 y_{b}^{2}-3 y_{t}^{2}+2 \sum_{g}\left(3 y_{u_{g}}^{2}+3 y_{d_{g}}^{2}+y_{e_{g}}^{2}\right)-\frac{1}{4} g_{1}^{2}-\frac{9}{4} g_{2}^{2}-8 g_{3}^{2} .
\end{aligned}
$$

(iii) Higgs sector:

$$
\begin{aligned}
(4 \pi)^{2} \beta_{\lambda}^{(1)}= & 12 \lambda^{2}+8 \lambda \sum_{g}\left(3 y_{u_{g}}^{2}+3 y_{d_{g}}^{2}+y_{e_{g}}^{2}\right)-9 \lambda\left(\frac{1}{5} g_{1}^{2}+g_{2}^{2}\right) \\
& -16 \sum_{g}\left(3 y_{u_{g}}^{4}+3 y_{d_{g}}^{4}+y_{e_{g}}^{4}\right)+\frac{9}{4}\left(\frac{3}{25} g_{1}^{4}+g_{2}^{4}+\frac{2}{5} g_{1}^{2} g_{2}^{2}\right), \\
(4 \pi)^{2} v^{-1} \gamma_{v}^{(1)=} & -2 \sum_{g}\left(3 y_{u_{g}}^{2}+3 y_{d_{g}}^{2}+y_{e_{g}}^{2}\right)+\frac{9}{4}\left(\frac{1}{5} g_{1}^{2}+g_{2}^{2}\right) .
\end{aligned}
$$




\section{Two-loop contributions}

(i) Gauge sector:

$$
\begin{aligned}
& (4 \pi)^{4} g_{1}^{-3} \beta_{g_{1}}^{(2)}=\frac{199}{50} g_{1}^{2}+\frac{27}{10} g_{2}^{2}+\frac{44}{5} g_{3}^{2}-\sum_{g}\left(\frac{17}{5} y_{u_{g}}^{2}+y_{d_{g}}^{2}+3 y_{e_{g}}^{2}\right) \\
& (4 \pi)^{4} g_{2}^{-3} \beta_{g_{2}}^{(2)}=\frac{9}{10} g_{1}^{2}+\frac{35}{6} g_{2}^{2}+12 g_{3}^{2}-\sum_{g}\left(3 y_{u_{g}}^{2}+3 y_{d_{g}}^{2}+y_{e_{g}}^{2}\right) \\
& (4 \pi)^{4} g_{3}^{-3} \beta_{g_{3}}^{(2)}=\frac{11}{10} g_{1}^{2}+\frac{9}{2} g_{2}^{2}-26 g_{3}^{2}-4 \sum_{g}\left(y_{u_{g}}^{2}+y_{d_{g}}^{2}\right) .
\end{aligned}
$$

(ii) Yukawa sector:

$$
\begin{aligned}
(4 \pi)^{4} y_{\tau}^{-1} \beta_{y_{\tau}}^{(2)}= & 6 y_{\tau}^{4}-9 y_{\tau}^{2} \sum_{g}\left(3 y_{u_{g}}^{2}+3 y_{d_{g}}^{2}+y_{e_{g}}^{2}\right)-9 \sum_{g}\left(3 y_{u_{g}}^{4}+3 y_{u_{g}}^{4}\right. \\
& \left.-\frac{2}{3} y_{u_{g}}^{2} y_{d_{g}}^{2}+y_{e_{g}}^{4}\right)+\frac{3}{2} \lambda^{2}-12 \lambda y_{\tau}^{2}+\left(\frac{387}{40} g_{1}^{2}+\frac{135}{8} g_{2}^{2}\right) y_{\tau}^{2} \\
& +5\left(\frac{17}{20} g_{1}^{2}+\frac{9}{4} g_{2}^{2}+8 g_{3}^{2}\right) \sum_{g} y_{u_{g}}^{2}+5\left(\frac{1}{4} g_{1}^{2}+\frac{9}{4} g_{2}^{2}+8 g_{3}^{2}\right) \sum_{g} y_{d_{g}}^{2} \\
& +\frac{15}{4}\left(g_{1}^{2}+g_{2}^{2}\right) \sum_{g} y_{e_{g}}^{2}+\frac{1371}{200} g_{1}^{4}+\frac{27}{20} g_{1}^{2} g_{2}^{2}-\frac{23}{4} g_{2}^{4}, \\
(4 \pi)^{4} y_{t}^{-1} \beta_{y_{t}(2)=}= & 6 y_{t}^{4}-5 y_{t}^{2} y_{b}^{2}+11 y_{b}^{4}+\left(5 y_{b}^{2}-9 y_{t}^{2}\right) \sum_{g}\left(3 y_{u_{g}}^{2}+3 y_{d_{g}}^{2}+y_{e_{g}}^{2}\right) \\
& -9 \sum_{g}\left(3 y_{u_{g}}^{4}+3 y_{d_{g}}^{4}-\frac{2}{3} y_{u_{g}}^{2} y_{d_{g}}^{2}+y_{e_{g}}^{4}\right)+\frac{3}{2} \lambda^{2}-4 \lambda\left(3 y_{t}^{2}+y_{b}^{2}\right) \\
& +\left(\frac{223}{40} g_{1}^{2}+\frac{135}{8} g_{2}^{2}+32 g_{3}^{2}\right) y_{t}^{2}-\left(\frac{43}{40} g_{1}^{2}-\frac{9}{8} g_{2}^{2}+32 g_{3}^{2}\right) y_{b}^{2} \\
& +5\left(\frac{17}{20} g_{1}^{2}+\frac{9}{4} g_{2}^{2}+8 g_{3}^{2}\right) \sum_{g} y_{u_{g}}^{2}+5\left(\frac{1}{4} g_{1}^{2}+\frac{9}{4} g_{2}^{2}+8 g_{3}^{2}\right) \sum_{g} y_{d_{g}}^{2} \\
& +\frac{15}{4}\left(g_{1}^{2}+g_{2}^{2}\right) \sum_{g} y_{e_{g}}^{2} \\
& +\frac{1187}{600} g_{1}^{4}-\frac{9}{20} g_{1}^{2} g_{2}^{2}-\frac{23}{4} g_{2}^{4}+\frac{19}{15} g_{1}^{2} g_{3}^{2}+9 g_{2}^{2} g_{3}^{2}-108 g_{3}^{4}, \\
& 6 y_{b}^{4}-5 y_{b}^{2} y_{t}^{2}+11 y_{t}^{4}+\left(5 y_{t}^{2}-9 y_{b}^{2}\right) \sum_{g}\left(3 y_{u_{g}}^{2}+3 y_{d_{g}}^{2}+y_{e_{g}}^{2}\right) \\
& -9 \sum_{g}\left(3 y_{u_{g}}^{4}+3 y_{d_{g}}^{4}-\frac{2}{3} y_{u_{g}}^{2} y_{d_{g}}^{2}+y_{e_{g}}^{4}\right)+\frac{3}{2} \lambda^{2}-4 \lambda\left(3 y_{b}^{2}+y_{t}^{2}\right) \\
& +\left(\frac{187}{40} g_{1}^{2}+\frac{135}{8} g_{2}^{2}+32 g_{3}^{2}\right) y_{b}^{2}-\left(\frac{79}{40} g_{1}^{2}-\frac{9}{8} g_{2}^{2}+32 g_{3}^{2}\right) y_{t}^{2} \\
& +5\left(\frac{17}{20} g_{1}^{2}+\frac{9}{4} g_{2}^{2}+8 g_{3}^{2}\right) \sum_{g} y_{u_{g}}^{2}+5\left(\frac{1}{4} g_{1}^{2}+\frac{9}{4} g_{2}^{2}+8 g_{3}^{2}\right) \sum_{g} y_{d_{g}}^{2} \\
& +\frac{15}{4}\left(g_{1}^{2}+g_{2}^{2}\right) \sum_{g} y_{e_{g}}^{2} \\
& -\frac{127}{600} g_{1}^{4}-\frac{27}{20} g_{1}^{2} g_{2}^{2}-\frac{23}{4} g_{2}^{4}+\frac{31}{15} g_{1}^{2} g_{3}^{2}+9 g_{2}^{2} g_{3}^{2}-108 g_{3}^{4} .
\end{aligned}
$$


(iii) Higgs sector:

$$
\begin{aligned}
(4 \pi)^{4} \beta_{\lambda}^{(2)=} & -78 \lambda^{3}+54 \lambda^{2}\left(\frac{1}{5} g_{1}^{2}+g_{2}^{2}\right)+\lambda\left(\frac{1887}{200} g_{1}^{4}+\frac{117}{20} g_{1}^{2} g_{2}^{2}-\frac{73}{8} g_{2}^{4}\right) \\
& -\frac{3411}{1000} g_{1}^{6}-\frac{1677}{200} g_{1}^{4} g_{2}^{2}-\frac{289}{40} g_{1}^{2} g_{2}^{4}+\frac{305}{8} g_{2}^{6} \\
& -3 g_{2}^{4} \sum_{g}\left(3 y_{u_{g}}^{2}+3 y_{d_{g}}^{2}+y_{e_{g}}^{2}\right) \\
& -\frac{32}{5} g_{1}^{2} \sum_{g}\left(2 y_{u_{g}}^{4}-y_{d_{g}}^{4}+3 y_{e_{g}}^{4}\right)-256 g_{3}^{2} \sum_{g}\left(y_{u_{g}}^{4}+y_{d_{g}}^{4}\right) \\
& +20 \lambda\left(\left(\frac{17}{20} g_{1}^{2}+\frac{9}{4} g_{2}^{2}+8 g_{3}^{2}\right) \sum_{g} y_{u_{g}}^{2}+\left(\frac{1}{4} g_{1}^{2}+\frac{9}{4} g_{2}^{2}+8 g_{3}^{2}\right) \sum_{g} y_{d_{g}}^{2}\right. \\
& \left.+\frac{3}{4}\left(g_{1}^{2}+g_{2}^{2}\right) \sum_{g} y_{e_{g}}^{2}\right)+\frac{6}{5} g_{1}^{2}\left(\left(-\frac{57}{10} g_{1}^{2}+21 g_{2}^{2}\right) \sum_{g} y_{u_{g}}^{2}\right. \\
& \left.+3\left(\frac{1}{2} g_{1}^{2}+3 g_{2}^{2}\right) \sum_{g} y_{d_{g}}^{2}+\left(-\frac{15}{2} g_{1}^{2}+11 g_{2}^{2}\right) \sum_{g} y_{e_{g}}^{2}\right) \\
& -48 \lambda^{2} \sum_{g}\left(3 y_{u_{g}}^{2}+3 y_{d_{g}}^{2}+y_{e_{g}}^{2}\right) \\
& -4 \lambda \sum_{g}\left(3 y_{u_{g}}^{4}+3 y_{d_{g}}^{4}+y_{e_{g}}^{4}-6 y_{u_{g}}^{2} y_{d_{g}}^{2}\right) \\
& +160 \sum_{g}\left(3 y_{u_{g}}^{6}+3 y_{d_{g}}^{6}+y_{e_{g}}^{6}\right)-96 \sum_{g}\left(y_{u_{g}}^{4} y_{d_{g}}^{2}+y_{d_{g}}^{4} y_{u_{g}}^{2}\right), \\
(4 \pi)^{4} v^{-1} \gamma_{v}^{(2)}= & -\frac{3}{2} \lambda^{2}-\frac{1293}{800} g_{1}^{4}+\frac{271}{32} g_{2}^{4}-\frac{27}{80} g_{1}^{2} g_{2}^{2} \\
& -\frac{5}{2}\left(\frac{17}{10} g_{1}^{2}+\frac{9}{2} g_{2}^{2}+16 g_{3}^{2}\right) \sum_{g} y_{u_{g}}^{2}-\frac{5}{2}\left(\frac{1}{2} g_{1}^{2}+\frac{9}{2} g_{2}^{2}+16 g_{3}^{2}\right) \sum_{g} y_{d_{g}}^{2} \\
& -\frac{15}{4}\left(g_{1}^{2}+g_{2}^{2}\right) \sum_{g} y_{e_{g}}^{2}+9 \sum_{g}\left(3 y_{u}^{4}+3 y_{d}^{4}-\frac{2}{3} y_{u}^{2} y_{d}^{2}+y_{e}^{4}\right) .
\end{aligned}
$$

\section{A2 Neutrino Yukawa contributions to SM $\beta$ functions}

\section{One-loop contributions}

(i) Yukawa sector:

$$
\begin{aligned}
(4 \pi)^{2} \mathbf{Y}_{\nu}^{-1} \beta_{\mathbf{Y}_{\nu}}^{(1)} & =\frac{3}{2}\left(\mathbf{Y}_{\nu}^{\dagger} \mathbf{Y}_{\nu}-\mathbf{Y}_{e}^{\dagger} \mathbf{Y}_{e}\right)+Y_{2}(S)-\frac{9}{20} g_{1}^{2}-\frac{9}{4} g_{2}^{2} \\
(4 \pi)^{2} \mathbf{Y}_{e}^{-1} \Delta \beta_{\mathbf{Y}_{e}}^{(1)} & =-\frac{3}{2} \mathbf{Y}_{\nu}^{\dagger} \mathbf{Y}_{\nu}+\operatorname{Tr}\left(\mathbf{Y}_{\nu}^{\dagger} \mathbf{Y}_{\nu}\right) \\
(4 \pi)^{2} \mathbf{Y}_{u}^{-1} \Delta \beta_{\mathbf{Y}_{u}}^{(1)} & =\operatorname{Tr}\left(\mathbf{Y}_{\nu}^{\dagger} \mathbf{Y}_{\nu}\right) \\
(4 \pi)^{2} \mathbf{Y}_{d}^{-1} \Delta \beta_{\mathbf{Y}_{d}}^{(1)} & =\operatorname{Tr}\left(\mathbf{Y}_{\nu}^{\dagger} \mathbf{Y}_{\nu}\right)
\end{aligned}
$$

(ii) Higgs sector:

$$
\begin{aligned}
(4 \pi)^{2} \Delta \beta_{\lambda}^{(1)} & =4 \lambda \operatorname{Tr}\left(\mathbf{Y}_{\nu}^{\dagger} \mathbf{Y}_{\nu}\right)-4 \operatorname{Tr}\left(\left(\mathbf{Y}_{\nu}^{\dagger} \mathbf{Y}_{\nu}\right)^{2}\right) \\
(4 \pi)^{2} v^{-1} \Delta \gamma_{v}^{(1)} & =-\operatorname{Tr}\left(\mathbf{Y}_{\nu}^{\dagger} \mathbf{Y}_{\nu}\right) .
\end{aligned}
$$




\section{Two-loop contributions ${ }^{7}$}

(i) Gauge sector:

$$
\begin{aligned}
& (4 \pi)^{4} g_{1}^{-3} \Delta \beta_{g_{1}}^{(2)}=-\frac{3}{10} \operatorname{Tr}\left(\mathbf{Y}_{\nu}^{\dagger} \mathbf{Y}_{\nu}\right) \\
& (4 \pi)^{4} g_{2}^{-3} \Delta \beta_{g_{2}}^{(2)}=-\frac{1}{2} \operatorname{Tr}\left(\mathbf{Y}_{\nu}^{\dagger} \mathbf{Y}_{\nu}\right)
\end{aligned}
$$

(ii) Yukawa sector:

$$
\begin{aligned}
(4 \pi)^{4} \mathbf{Y}_{\nu}^{-1} \beta_{\mathbf{Y}_{\nu}}^{(2)}= & \frac{3}{2}\left(\mathbf{Y}_{\nu}^{\dagger} \mathbf{Y}_{\nu}\right)^{2}-\mathbf{Y}_{\nu}^{\dagger} \mathbf{Y}_{\nu} \mathbf{Y}_{e}^{\dagger} \mathbf{Y}_{e}-\frac{1}{4} \mathbf{Y}_{e}^{\dagger} \mathbf{Y}_{e} \mathbf{Y}_{\nu}^{\dagger} \mathbf{Y}_{\nu}+\frac{11}{4}\left(\mathbf{Y}_{e}^{\dagger} \mathbf{Y}_{e}\right)^{2} \\
& +Y_{2}(S)\left(\frac{5}{4} \mathbf{Y}_{e}^{\dagger} \mathbf{Y}_{e}-\frac{9}{4} \mathbf{Y}_{\nu}^{\dagger} \mathbf{Y}_{\nu}\right)-\chi_{4}(S) \\
& +\frac{3}{2} \lambda^{2}-2 \lambda\left(3 \mathbf{Y}_{\nu}^{\dagger} \mathbf{Y}_{\nu}+\mathbf{Y}_{e}^{\dagger} \mathbf{Y}_{e}\right) \\
& +\left(\frac{279}{80} g_{1}^{2}+\frac{135}{16} g_{2}^{2}\right) \mathbf{Y}_{\nu}^{\dagger} \mathbf{Y}_{\nu}-\left(\frac{243}{80} g_{1}^{2}-\frac{9}{16} g_{2}^{2}\right) \mathbf{Y}_{e}^{\dagger} \mathbf{Y}_{e}+\frac{5}{2} Y_{4}(S) \\
& +\left(-\frac{3}{40}+\frac{1}{5} n_{g}\right) g_{1}^{4}-\frac{27}{20} g_{1}^{2} g_{2}^{2}-\left(\frac{35}{4}-n_{g}\right) g_{2}^{4}, \\
(4 \pi)^{4} \mathbf{Y}_{e}^{-1} \Delta \beta_{\mathbf{Y}_{e}}^{(2)}= & -\mathbf{Y}_{e}^{\dagger} \mathbf{Y}_{e} \mathbf{Y}_{\nu}^{\dagger} \mathbf{Y}_{\nu}-\frac{1}{4} \mathbf{Y}_{\nu}^{\dagger} \mathbf{Y}_{\nu} \mathbf{Y}_{e}^{\dagger} \mathbf{Y}_{e}+\frac{11}{4}\left(\mathbf{Y}_{\nu}^{\dagger} \mathbf{Y}_{\nu}\right)^{2} \\
& +\frac{5}{4} Y_{2}(S) \mathbf{Y}_{\nu}^{\dagger} \mathbf{Y}_{\nu}-2 \lambda \mathbf{Y}_{\nu}^{\dagger} \mathbf{Y}_{\nu}+\left(-\frac{27}{16} g_{1}^{2}+\frac{9}{16} g_{2}^{2}\right) \mathbf{Y}_{\nu}^{\dagger} \mathbf{Y}_{\nu}, \\
(4 \pi)^{4} \mathbf{Y}_{u}^{-1} \Delta \beta_{\mathbf{Y}_{u}}^{(2)}= & \left(\frac{5}{4} \mathbf{Y}_{d}^{\dagger} \mathbf{Y}_{d}-\frac{9}{4} \mathbf{Y}_{u}^{\dagger} \mathbf{Y}_{u}\right) \operatorname{Tr}\left(\mathbf{Y}_{\nu}^{\dagger} \mathbf{Y}_{\nu}\right)-\operatorname{Tr}\left(\frac{9}{4}\left(\mathbf{Y}_{\nu}^{\dagger} \mathbf{Y}_{\nu}\right)^{2}\right. \\
& \left.-\frac{1}{2} \mathbf{Y}_{\nu}^{\dagger} \mathbf{Y}_{\nu} \mathbf{Y}_{e}^{\dagger} \mathbf{Y}_{e}\right)+\frac{15}{8}\left(\frac{1}{5} g_{1}^{2}+g_{2}^{2}\right) \operatorname{Tr}\left(\mathbf{Y}_{\nu}^{\dagger} \mathbf{Y}_{\nu}\right) \\
(4 \pi)^{4} \mathbf{Y}_{d}^{-1} \Delta \beta_{\mathbf{Y}_{d}}^{(2)}= & \left(\frac{5}{4} \mathbf{Y}_{u}^{\dagger} \mathbf{Y}_{u}-\frac{9}{4} \mathbf{Y}_{d}^{\dagger} \mathbf{Y}_{d}\right) \operatorname{Tr}\left(\mathbf{Y}_{\nu}^{\dagger} \mathbf{Y}_{\nu}\right)-\operatorname{Tr}\left(\frac{9}{4}\left(\mathbf{Y}_{\nu}^{\dagger} \mathbf{Y}_{\nu}\right)^{2}\right. \\
& \left.-\frac{1}{2} \mathbf{Y}_{\nu}^{\dagger} \mathbf{Y}_{\nu} \mathbf{Y}_{e}^{\dagger} \mathbf{Y}_{e}\right)+\frac{15}{8}\left(\frac{1}{5} g_{1}^{2}+g_{2}^{2}\right) \operatorname{Tr}\left(\mathbf{Y}_{\nu}^{\dagger} \mathbf{Y}_{\nu}\right)
\end{aligned}
$$

(iii) Higgs sector:

$$
\begin{aligned}
(4 \pi)^{4} \Delta \beta_{\lambda}^{(2)}= & -\frac{3}{2} g_{2}^{4} \operatorname{Tr}\left(\mathbf{Y}_{\nu}^{\dagger} \mathbf{Y}_{\nu}\right)-\frac{3}{10} g_{1}^{2}\left(\frac{3}{5} g_{1}^{2}+2 g_{2}^{2}\right) \operatorname{Tr}\left(\mathbf{Y}_{\nu}^{\dagger} \mathbf{Y}_{\nu}\right) \\
& +\frac{15}{2} \lambda\left(\frac{1}{5} g_{1}^{2}+g_{2}^{2}\right) \operatorname{Tr}\left(\mathbf{Y}_{\nu}^{\dagger} \mathbf{Y}_{\nu}\right)-24 \lambda^{2} \operatorname{Tr}\left(\mathbf{Y}_{\nu}^{\dagger} \mathbf{Y}_{\nu}\right) \\
& -\lambda \operatorname{Tr}\left(\left(\mathbf{Y}_{\nu}^{\dagger} \mathbf{Y}_{\nu}\right)^{2}\right)+2 \lambda \operatorname{Tr}\left(\mathbf{Y}_{\nu}^{\dagger} \mathbf{Y}_{\nu} \mathbf{Y}_{e}^{\dagger} \mathbf{Y}_{e}\right) \\
& +20 \operatorname{Tr}\left(\left(\mathbf{Y}_{\nu}^{\dagger} \mathbf{Y}_{\nu}\right)^{3}\right) \\
& -4 \operatorname{Tr}\left(\mathbf{Y}_{\nu}^{\dagger} \mathbf{Y}_{\nu}\left(\mathbf{Y}_{\nu}^{\dagger} \mathbf{Y}_{\nu}+\mathbf{Y}_{e}^{\dagger} \mathbf{Y}_{e}\right) \mathbf{Y}_{e}^{\dagger} \mathbf{Y}_{e}\right), \\
(4 \pi)^{2} v^{-1} \Delta \gamma_{v}^{(2)}= & -\frac{15}{8} \operatorname{Tr}\left(\left(\frac{1}{5} g_{1}^{2}+g_{2}^{2}\right) \mathbf{Y}_{\nu}^{\dagger} \mathbf{Y}_{\nu}\right) \\
& +\frac{9}{4} \operatorname{Tr}\left(\left(\mathbf{Y}_{\nu}^{\dagger} \mathbf{Y}_{\nu}\right)^{2}\right)-\frac{1}{2} \operatorname{Tr}\left(\mathbf{Y}_{\nu}^{\dagger} \mathbf{Y}_{\nu} \mathbf{Y}_{e}^{\dagger} \mathbf{Y}_{e}\right),
\end{aligned}
$$

${ }^{7}$ The expression for $\beta_{N}^{(2)}$ is complete. For the other $\beta$-functions, neutrino induced contributions included into $Y_{2}(S), Y_{4}(S)$ and $\chi_{4}(S)$ invariants are omitted. 
where

$$
\begin{aligned}
Y_{2}(S)= & \operatorname{Tr}\left(3 \mathbf{Y}_{u}^{\dagger} \mathbf{Y}_{u}+3 \mathbf{Y}_{d}^{\dagger} \mathbf{Y}_{d}+\mathbf{Y}_{\nu}^{\dagger} \mathbf{Y}_{\nu}+\mathbf{Y}_{e}^{\dagger} \mathbf{Y}_{e}\right) \\
\chi_{4}(S)= & \frac{9}{4} \operatorname{Tr}\left(3\left(\mathbf{Y}_{u}^{\dagger} \mathbf{Y}_{u}\right)^{2}+3\left(\mathbf{Y}_{d}^{\dagger} \mathbf{Y}_{d}\right)^{2}+\left(\mathbf{Y}_{\nu}^{\dagger} \mathbf{Y}_{\nu}\right)^{2}\right. \\
& \left.+\left(\mathbf{Y}_{e}^{\dagger} \mathbf{Y}_{e}\right)^{2}-\frac{2}{3} \mathbf{Y}_{u}^{\dagger} \mathbf{Y}_{u} \mathbf{Y}_{d}^{\dagger} \mathbf{Y}_{d}-\frac{2}{9} \mathbf{Y}_{\nu}^{\dagger} \mathbf{Y}_{\nu} \mathbf{Y}_{e}^{\dagger} \mathbf{Y}_{e}\right) \\
Y_{4}(S)= & \operatorname{Tr}\left(\left(\frac{17}{20} g_{1}^{2}+\frac{9}{4} g_{2}^{2}+8 g_{3}^{2}\right) \mathbf{Y}_{u}^{\dagger} \mathbf{Y}_{u}\right. \\
& +\left(\frac{1}{4} g_{1}^{2}+\frac{9}{4} g_{2}^{2}+8 g_{3}^{2}\right) \mathbf{Y}_{d}^{\dagger} \mathbf{Y}_{d} \\
& \left.+\frac{3}{4}\left(\frac{1}{5} g_{1}^{2}+g_{2}^{2}\right) \mathbf{Y}_{\nu}^{\dagger} \mathbf{Y}_{\nu}+\frac{3}{4}\left(g_{1}^{2}+g_{2}^{2}\right) \mathbf{Y}_{e}^{\dagger} \mathbf{Y}_{e}\right)
\end{aligned}
$$

Our definition of invariants generalizes immediately that of Ref. [13].

\section{A3 Heavy neutrino contributions to SM $\beta$ functions}

We put here for simplicity $\nu_{4}=N, e_{4}=E$. In what follows $n_{g}=4$ and the generation index $g$ runs over the values $g=1, \ldots, 4$. Our normalization for the Yukawa couplings $y_{f_{g}}$ corresponds to $\left(\mathbf{Y}_{f}^{\text {diag }}\right)_{g g^{\prime}}=\sqrt{2} y_{f_{g}} \delta_{g g^{\prime}}$. In practice, one has $y_{\nu_{g}}=0$ for $g \neq 4$.

\section{One-loop contributions}

(i) Yukawa sector:

$$
\begin{aligned}
(4 \pi)^{2} y_{N}^{-1} \beta_{y_{N}}^{(1)} & =3 y_{N}^{2}-3 y_{E}^{2}+Y_{2}(S)-\frac{9}{20} g_{1}^{2}-\frac{9}{4} g_{2}^{2}, \\
(4 \pi)^{2} y_{E}^{-1} \Delta \beta_{y_{E}}^{(1)} & =-y_{N}^{2}, \\
(4 \pi)^{2} y_{f}^{-1} \Delta \beta_{y_{f}}^{(1)} & =2 y_{N}^{2},
\end{aligned}
$$

where $f \neq N, E$.

(ii) Higgs sector:

$$
\begin{aligned}
(4 \pi)^{2} \Delta \beta_{\lambda}^{(1)} & =8 \lambda y_{N}^{2}-16 y_{N}^{4} . \\
(4 \pi)^{2} v^{-1} \Delta \gamma_{v}^{(1)} & =-2 y_{N}^{2} .
\end{aligned}
$$




\section{Two-loop contributions ${ }^{8}$}

(i) Gauge sector:

$$
\begin{aligned}
& (4 \pi)^{4} g_{1}^{-3} \Delta \beta_{g_{1}}^{(2)}=-\frac{3}{5} y_{N}^{2} \\
& (4 \pi)^{4} g_{2}^{-3} \Delta \beta_{g_{2}}^{(2)}=-y_{N}^{2}
\end{aligned}
$$

(ii) Yukawa sector:

$$
\begin{aligned}
(4 \pi)^{4} y_{N}^{-1} \beta_{y_{N}}^{(2)}= & 6 y_{N}^{4}-5 y_{E}^{2} y_{N}^{2}+11 y_{E}^{4}+\frac{1}{2} Y_{2}(S)\left(5 y_{E}^{2}-9 y_{N}^{2}\right) \\
& -\chi_{4}(S)+\frac{3}{2} \lambda^{2}-4 \lambda\left(3 y_{N}^{2}+y_{E}^{2}\right) \\
& +\left(\frac{279}{40} g_{1}^{2}+\frac{135}{8} g_{2}^{2}\right) y_{N}^{2}-\left(\frac{243}{40} g_{1}^{2}-\frac{9}{8} g_{2}^{2}\right) y_{E}^{2}+\frac{5}{2} Y_{4}(S) \\
& +\frac{3}{5}\left(-\frac{1}{8}+\frac{1}{3} n_{g}\right) g_{1}^{4}-\frac{27}{20} g_{1}^{2} g_{2}^{2}-\left(\frac{35}{4}-n_{g}\right) g_{2}^{4}, \\
(4 \pi)^{4} y_{E}^{-1} \Delta \beta_{y_{E}}^{(2)}= & 11 y_{N}^{4}-5 y_{E}^{2} y_{N}^{2}+\frac{5}{2} Y_{2}(S) y_{N}^{2} \\
& -4 \lambda y_{N}^{2}-\left(\frac{27}{8} g_{1}^{2}-\frac{9}{8} g_{2}^{2}\right) y_{N}^{2}, \\
(4 \pi)^{4} y_{u}^{-1} \Delta \beta_{y_{u}}^{(2)}= & \left(5 y_{d}^{2}-9 y_{u}^{2}\right) y_{N}^{2}-\left(9 y_{N}^{2}-2 y_{E}^{2}\right) y_{N}^{2}+\frac{15}{4}\left(\frac{1}{5} g_{1}^{2}+g_{2}^{2}\right) y_{N}^{2}, \\
(4 \pi)^{4} y_{d}^{-1} \Delta \beta_{y_{d}}^{(2)}= & \left(5 y_{u}^{2}-9 y_{d}^{2}\right) y_{N}^{2}-\left(9 y_{N}^{2}-2 y_{E}^{2}\right) y_{N}^{2}+\frac{15}{4}\left(\frac{1}{5} g_{1}^{2}+g_{2}^{2}\right) y_{N}^{2} .
\end{aligned}
$$

(iii) Higgs sector:

$$
\begin{aligned}
(4 \pi)^{4} \Delta \beta_{\lambda}^{(2)}= & -3 g_{2}^{4} y_{N}^{2}-\frac{3}{5} g_{1}^{2}\left(\frac{3}{5} g_{1}^{2}+2 g_{2}^{2}\right) y_{N}^{2} \\
& +15 \lambda\left(\frac{1}{5} g_{1}^{2}+g_{2}^{2}\right) y_{N}^{2}-48 \lambda^{2} y_{N}^{2} \\
& -4 \lambda y_{N}^{4}+8 \lambda y_{E}^{2} y_{N}^{2}+160 y_{N}^{6}-32 y_{E}^{2}\left(y_{N}^{2}+y_{E}^{2}\right) y_{N}^{2}, \\
(4 \pi)^{2} v^{-1} \Delta \gamma_{v}^{(2)}= & -\frac{15}{4}\left(\frac{1}{5} g_{1}^{2}+g_{2}^{2}\right) y_{N}^{2}+9 y_{N}^{4}-2 y_{N}^{2} y_{E}^{2}, \\
\text { where } & \\
Y_{2}(S)= & 2 \sum_{g}\left(3 y_{u_{g}}^{2}+3 y_{d_{g}}^{2}+y_{\nu_{g}}^{2}+y_{e_{g}}^{2}\right), \\
\chi_{4}(S)= & 9 \sum_{g}\left(3 y_{u_{g}}^{4}+3 y_{d_{g}}^{4}-\frac{2}{3} y_{u_{g}}^{2} y_{d_{g}}^{2}+y_{\nu_{g}}^{4}+y_{e_{g}}^{4}-\frac{2}{9} y_{\nu_{g}}^{2} y_{e_{g}}^{2}\right), \\
Y_{4}(S)= & 2 \sum_{g}\left(\left(\frac{17}{20} g_{1}^{2}+\frac{9}{4} g_{2}^{2}+8 g_{3}^{2}\right) y_{u_{g}}^{2}+\left(\frac{1}{4} g_{1}^{2}+\frac{9}{4} g_{2}^{2}+8 g_{3}^{2}\right) y_{d_{g}}^{2}\right. \\
& \left.+\frac{3}{4}\left(\frac{1}{5} g_{1}^{2}+g_{2}^{2}\right) y_{\nu_{g}}^{2}+\frac{3}{4}\left(g_{1}^{2}+g_{2}^{2}\right) y_{e_{g}}^{2}\right) .
\end{aligned}
$$

In fact only the third and fourth generations contribute here in the sums.

${ }^{8}$ The expression for $\beta_{N}^{(2)}$ is complete. For the other $\beta$-functions, neutrino induced contributions included into $Y_{2}(S), Y_{4}(S)$ and $\chi_{4}(S)$ invariants are omitted (cf. Appendix A2). 


\section{References}

[1] A. Peterman, Phys. Reports 53, 157 (1979).

[2] A. A. Vladimirov and D. V. Shirkov, Usp. Fiz. Nauk 129, 407 (1979) [Sov. Phys. Usp. 22, 860 (1979)].

[3] M. Lindner, Z. Phys. C - Part. and Fields 31, 295 (1986).

[4] D. J. E. Callaway and R. Petronzio, Nucl. Phys. B240, 577 (1984).

[5] M. Sher, Phys. Rep. 179, 273 (1989).

[6] N. Cabibbo, L. Maiani, G. Parisi and R. Petronzio, Nucl. Phys. B158, 295 (1979).

[7] M. A. B. Beg, C. Panagiotakopoulos and A. Sirlin, Phys. Rev. Lett. 52, 883 (1984).

[8] B. Grzadkowski and M. Lindner, Phys. Lett. B178, 81 (1986).

[9] H. Arason et. al., Phys. Rev. D47, 232 (1992).

[10] J. S. Lee and J. K. Kim, Phys. Rev. D53, 6689 (1996), hep-ph/9602406.

[11] H. B. Nielsen, A. V. Novikov, V. A. Novikov and M. I. Vysotsky, Phys. Lett. B374, 127 (1996); V. Novikov, hep-ph/9606318.

[12] H. Zheng, preprint PSI-PR-96-07 (1996), hep-ph/9602340.

[13] M. E. Machacek and M. T. Vaughn, Nucl. Phys. B222, 83 (1983); ibid. B236, 221 (1984); ibid. B249, 70 (1985).

[14] C. Ford, I. Jack and D. R. I. Jones, Nucl. Phys. B387, 373 (1992).

[15] C. Ford, D. R. I. Jones, P. W. Stephenson and M. B. Einhorn, Nucl. Phys. B395, 17 (1993).

[16] V. Barger, M. S. Berger and P. Ohmann, Phys. Rev. D47, 1093 (1993).

[17] B. Schrempp and M. Wimmer, Prog. Part. Nucl. Phys. 37, 1 (1996), hep-ph/9606386.

[18] R. M. Barnett et al., Phys. Rev. D54, 1 (1996).

[19] R. Hempfling and B. Kniel, Phys. Rev. D51, 1386 (1995).

[20] A. Sirlin and A. Zicchini, Nucl. Phys. B266, 389 (1986). 
[21] CDF Collaboration, F. Abe et al., FERMILAB-Pub-97/284-E (1997), FERMILAB-Pub-97/304-E (1997); D0 Collaboration, S. Abachi et al., Phys. Rev. Lett. 79, 1197 (1997), B. Abbot et al., FERMILAB-Pub97/172-E (1997); CDF and D0 Collaborations, S.R. Blusk, FermilabConf-98-151-E (1998).

[22] E. Tournefier (ALEPH Collaboration), Report at the Int. Seminar "Quarks '98", Suzdal, May 17-24, 1998.

[23] A. A. Vladimirov, D. I. Kazakov and O. V. Tarasov, Sov. Phys. JETP 50, 521 (1979); I. Jack and H. Osborn, J. Phys. A16, 1101 (1983).

[24] G. Altarelli and G. Isidori, Phys. Lett. B337, 141 (1994).

[25] J. A. Casas, J. R. Espinosa, M. Quiros and A. Riotto, Nucl. Phys. B436, 3 (1994); Erratum ibid. B439, 466 (1995); J. A. Casas, J. R. Espinosa and M. Quiros, Phys. Lett. B324, 171 (1995); ibid. B353, 54 (1995).

[26] CDF Collaboration, F. Abe et al., Phys. Rev. D46, 1989 (1992).

[27] OPAL Collaboration, CERN-EP/98-039 (1998). 


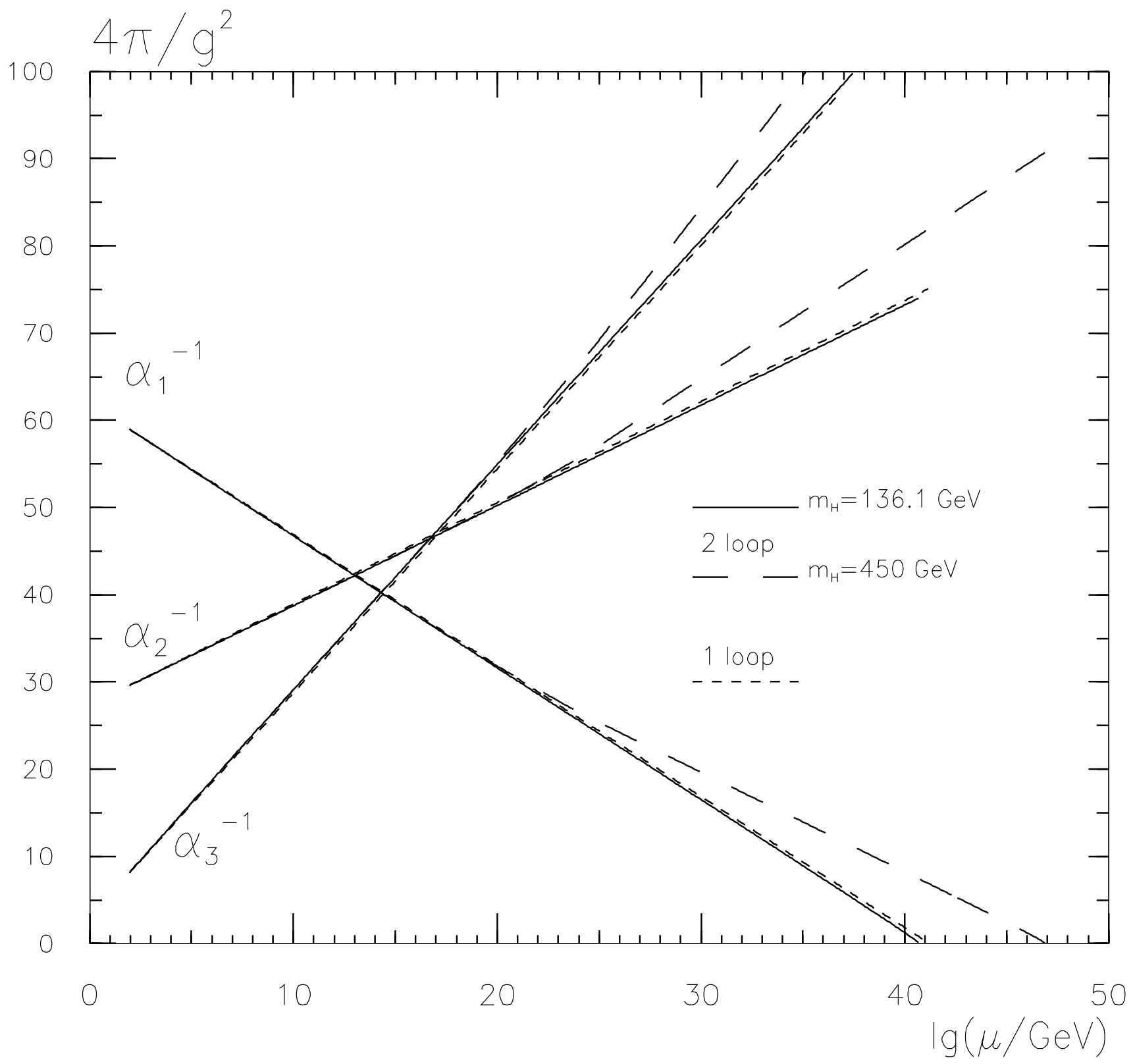

Fig. 1: Running of the inverse gauge couplings squared $\alpha_{i}^{-1}, i=1,2,3$. Number of generations is $n_{g}=3$. Represented Higgs masses are those corresponding to the typical heavy Higgs and to the lower critical Higgs curve shown in bold in Fig. 3. 


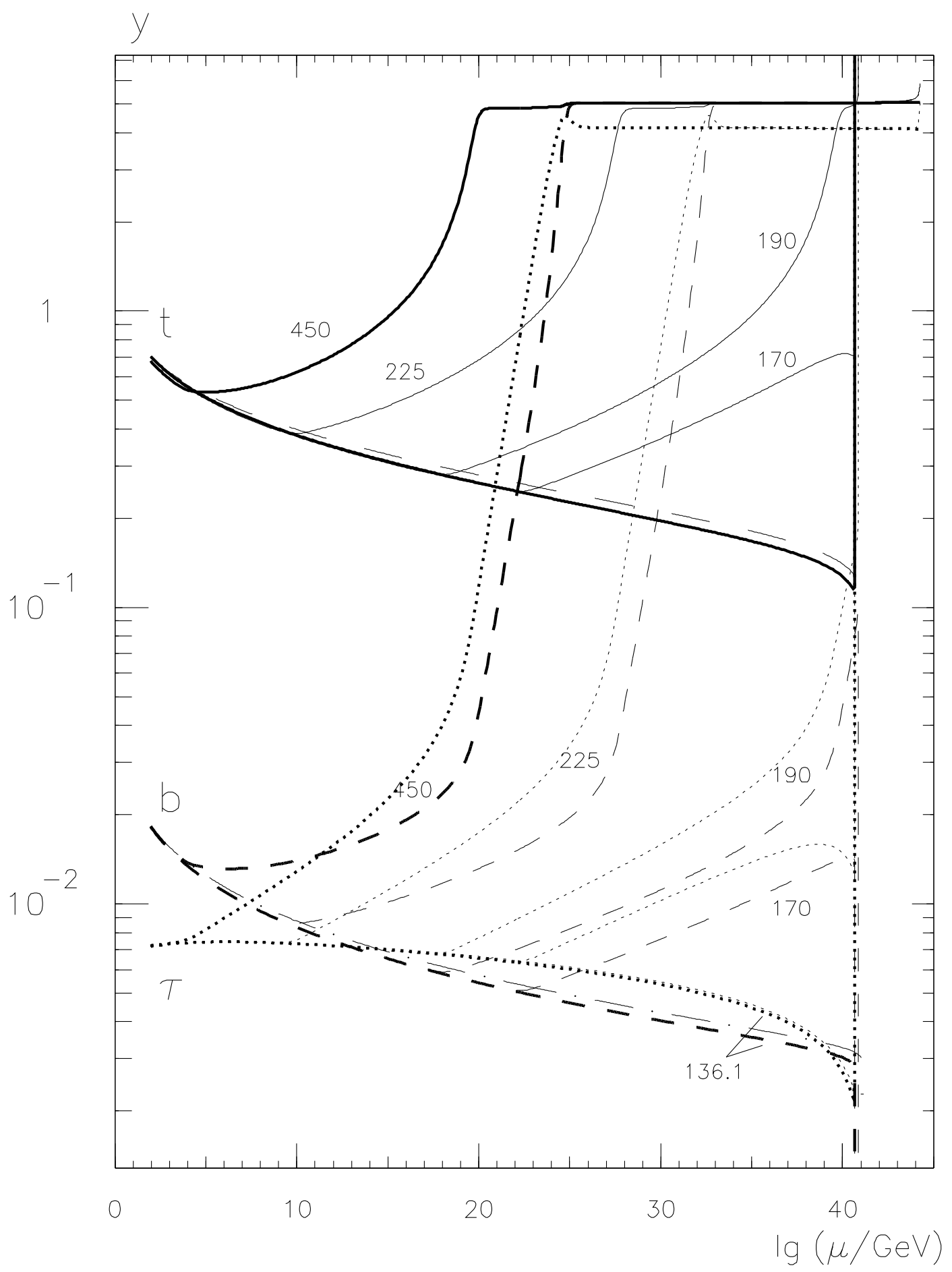

Fig. 2: Running of the third family Yukawa couplings $\left(n_{g}=3\right)$. The falling down curves shown in bold correspond to the lower critical Higgs mass. The thin lines, close to the latter bold ones, correspond to the oneloop approximation. 


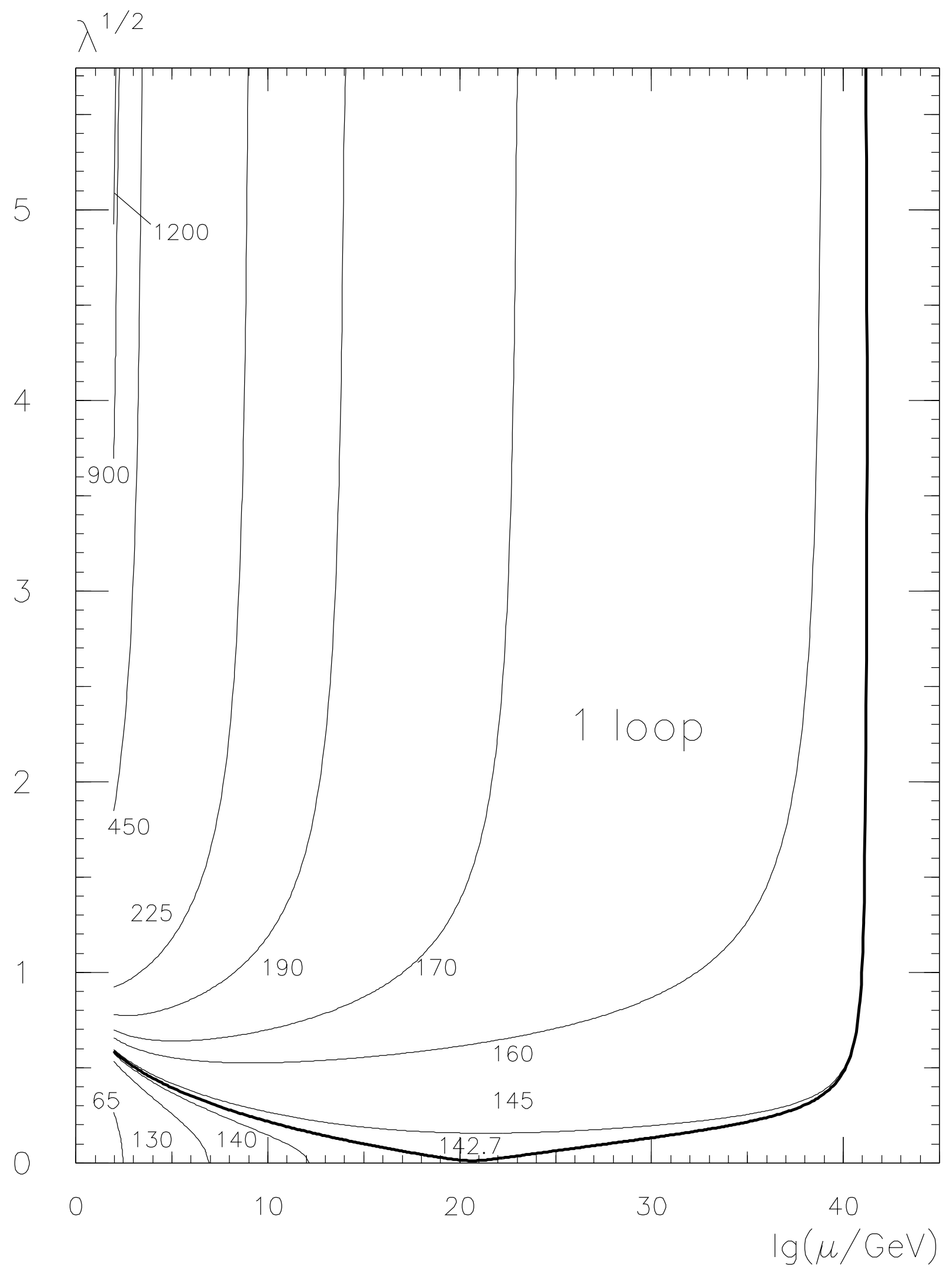

Fig. 3a: Running of the Higgs quartic coupling $\left(n_{g}=3\right)$ in one loop. The critical curves are shown in bold. 


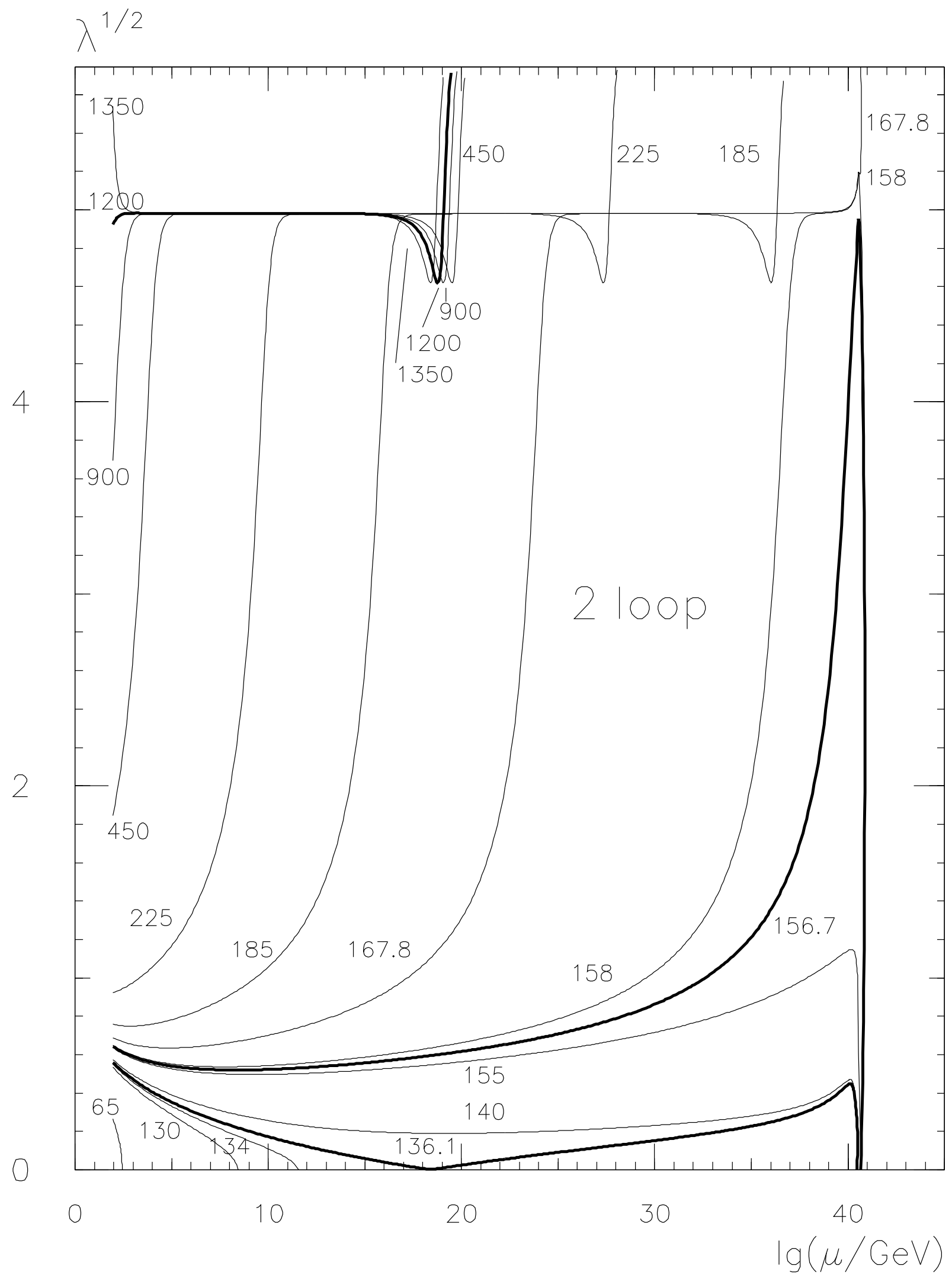

Fig. 3b: Running of the Higgs quartic coupling $\left(n_{g}=3\right)$ in two loops. The rest is as in Fig. 3a. 


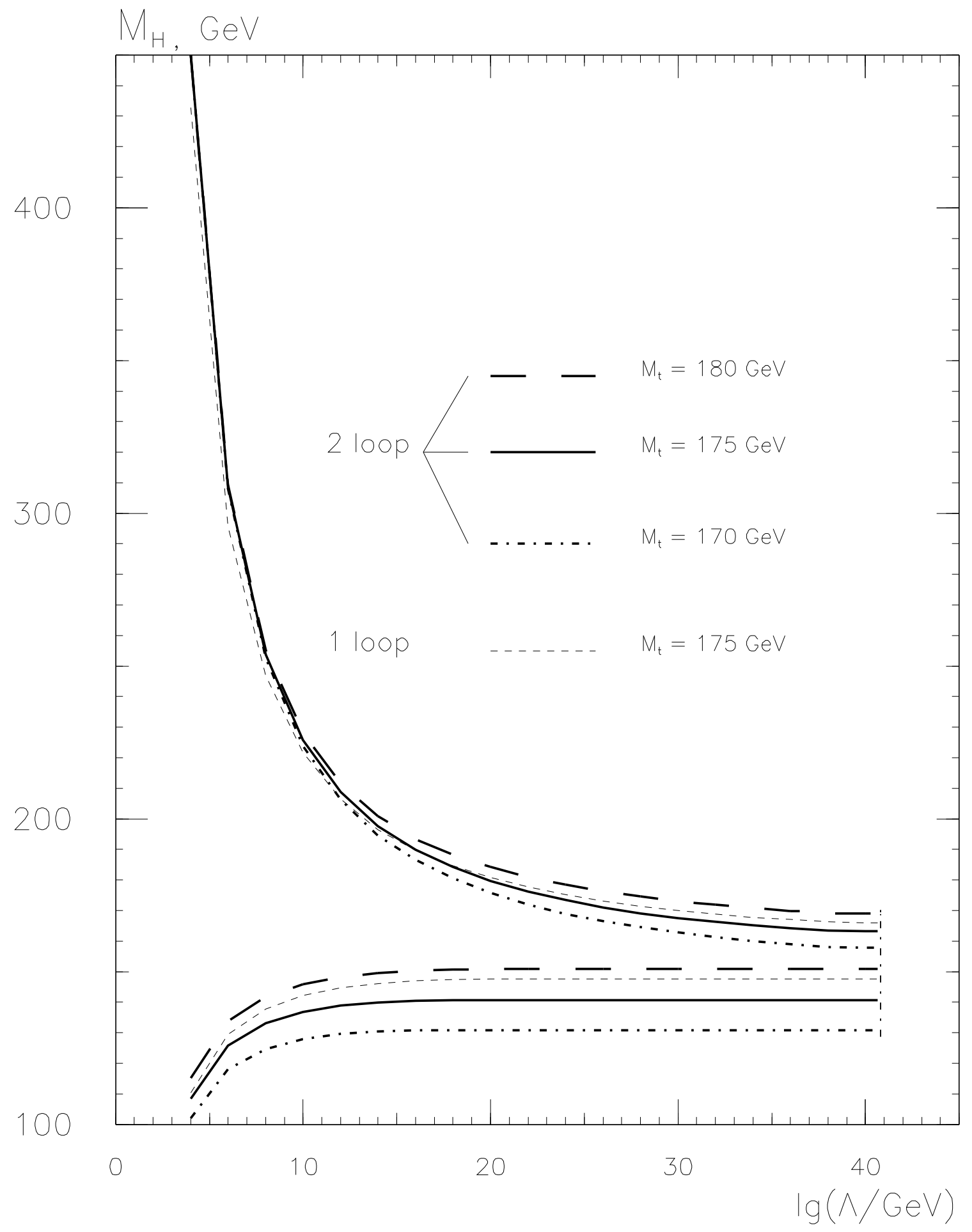

Fig. 4: The SM one- and two-loop self-consistency plot $\left(n_{g}=3\right)$ : the allowed Higgs mass vs. the cutoff scale $\Lambda$. 


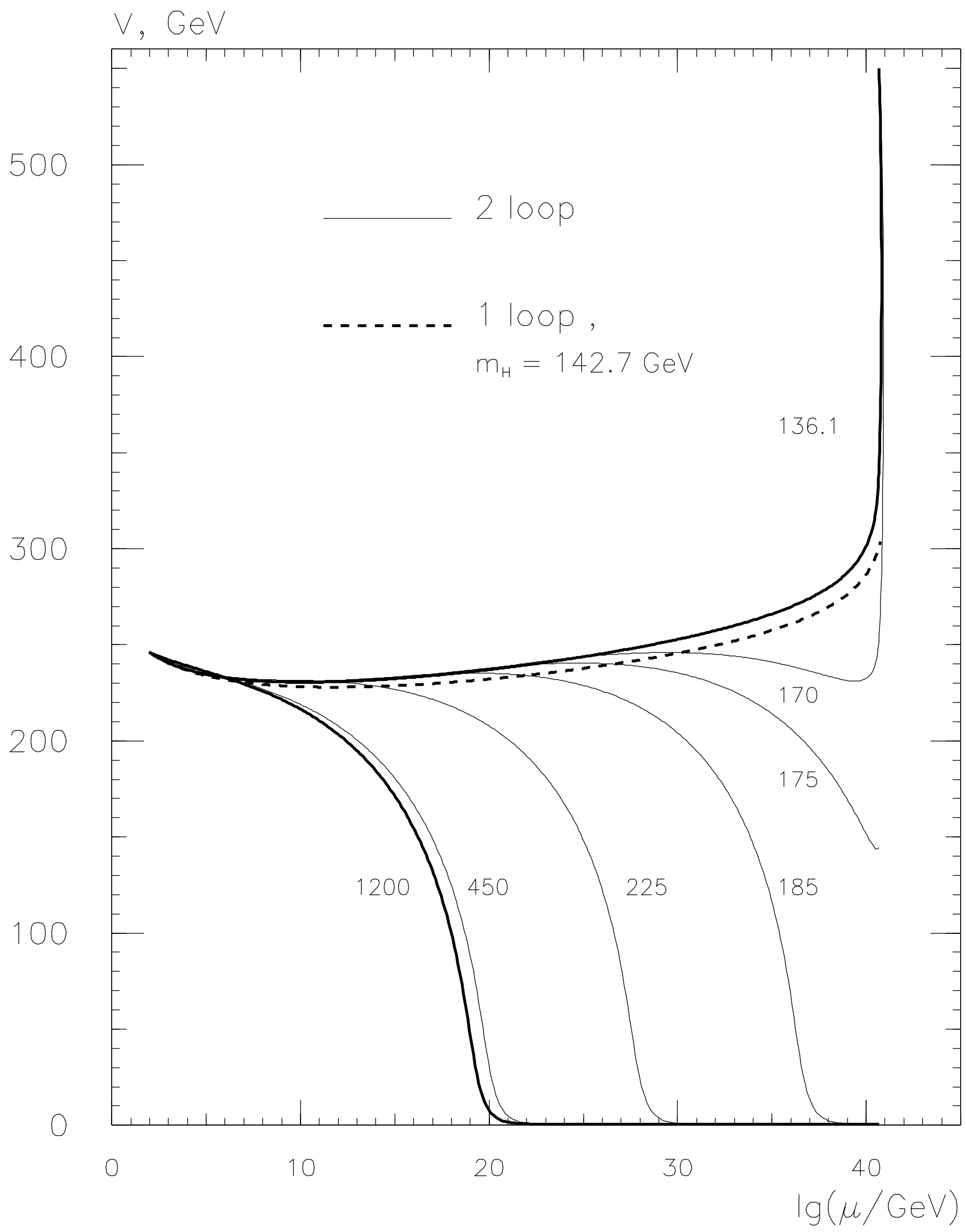

Fig. 5: Running of the Higgs VEV $\left(n_{g}=3\right)$ in the 't Hooft-Landau gauge. 


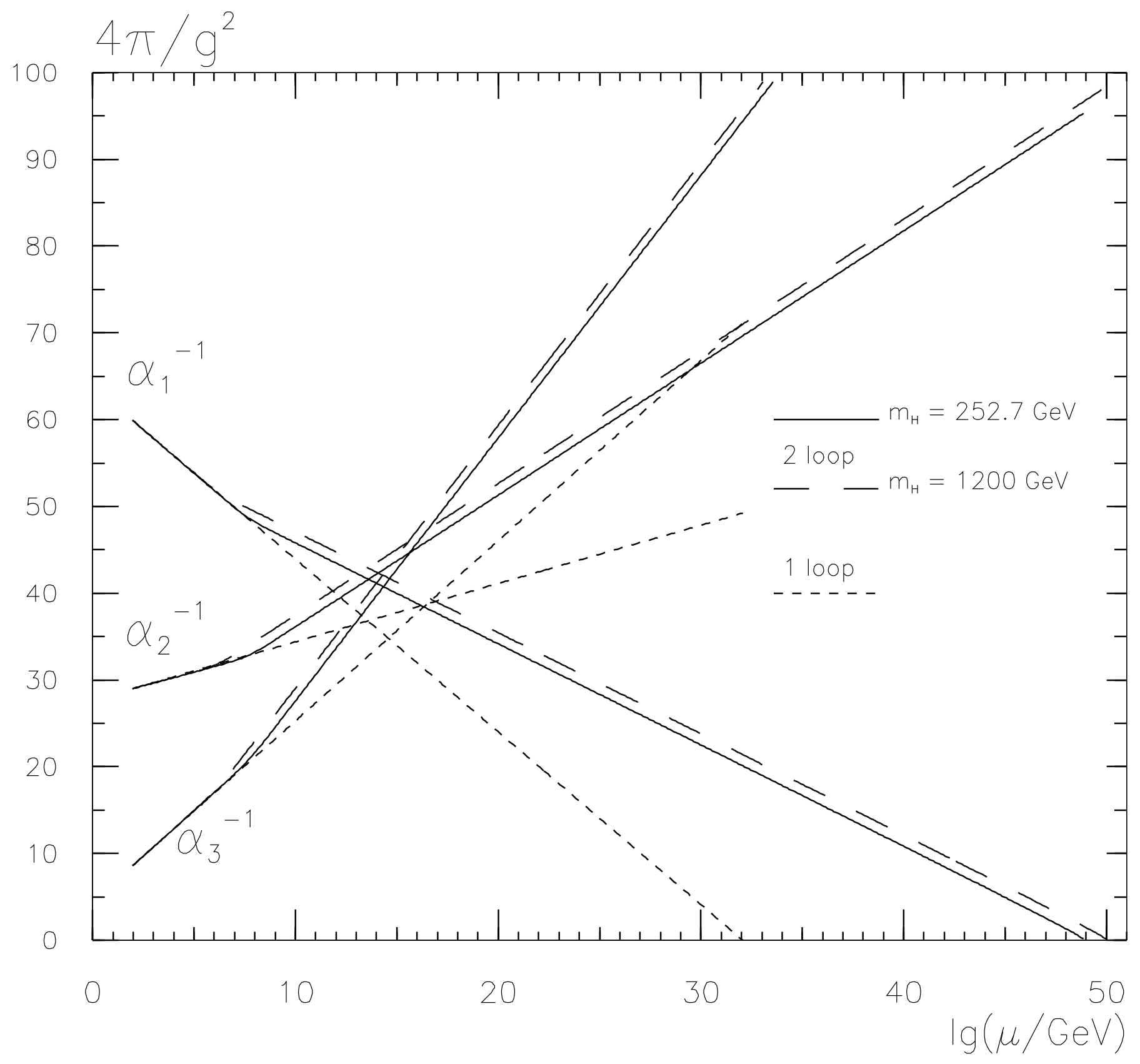

Fig. 6a: Running of the gauge couplings $\left(n_{g}=4\right)$. The fourth family mass scale is $m_{4}=200 \mathrm{GeV}$ and $m_{L} / m_{Q}=1 / 2$. 


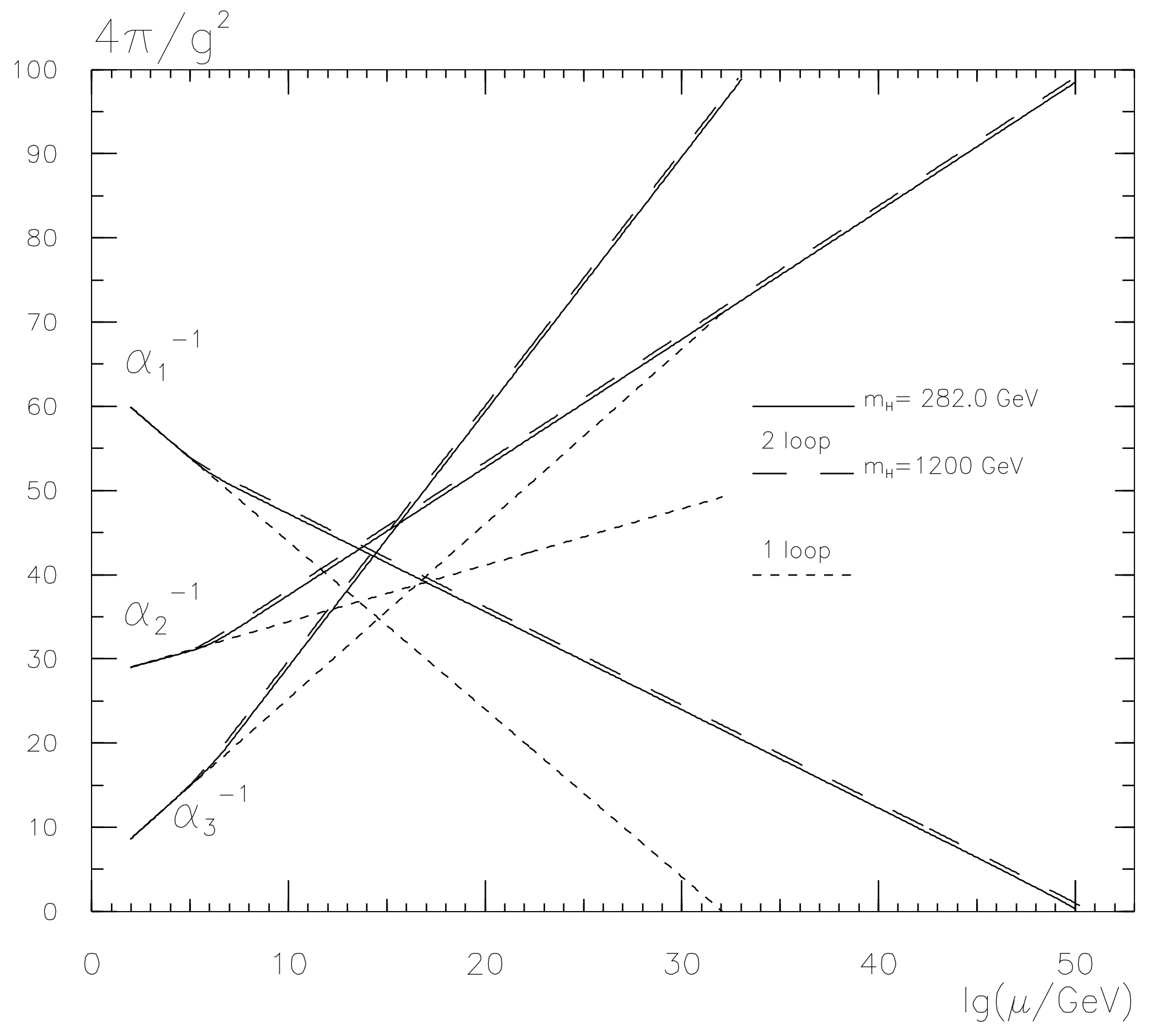

Fig. 6b: The same as in Fig. 6a at $m_{L} / m_{Q}=1$. 


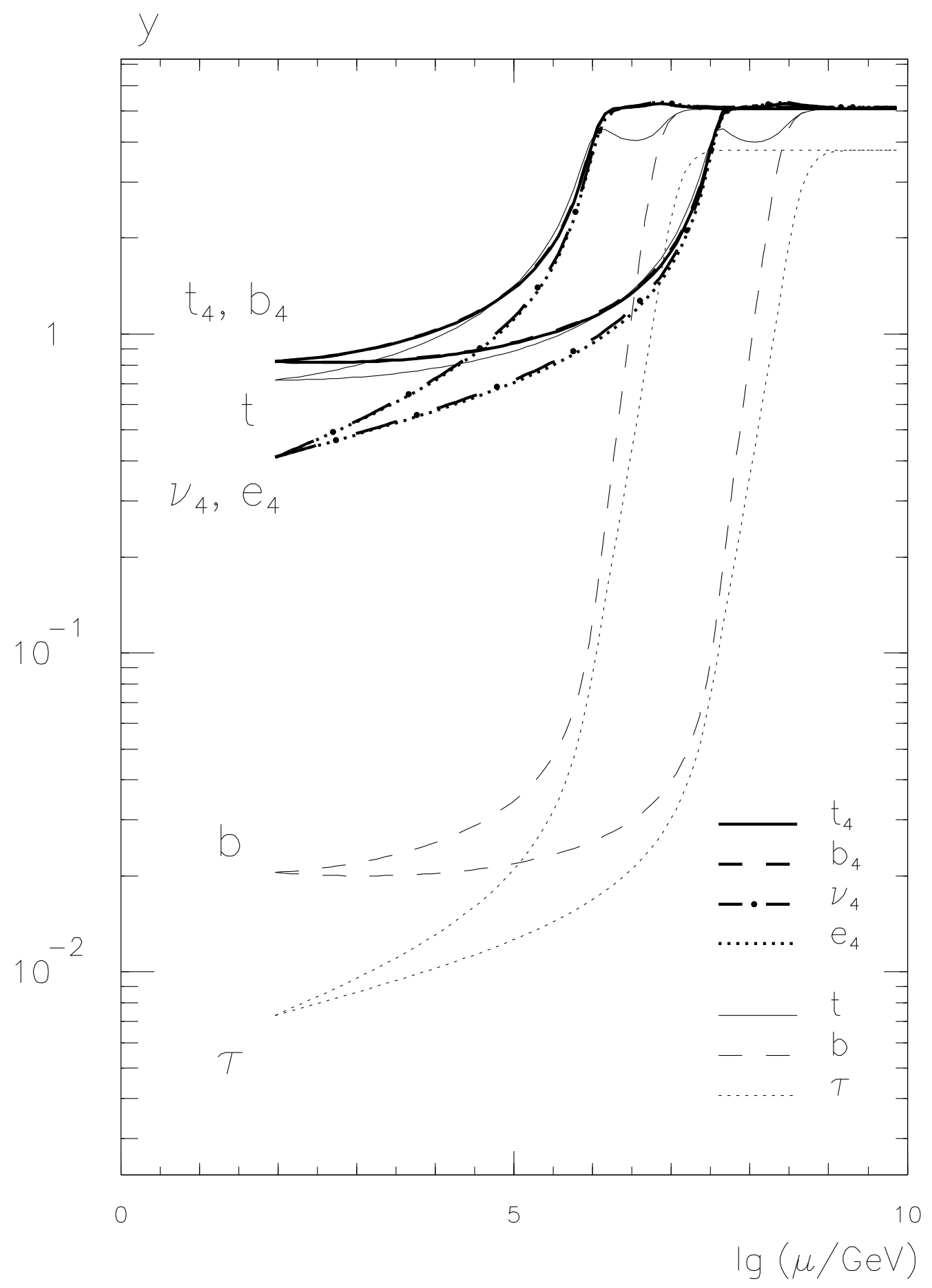

Fig. 7a: Two-loop running of the Yukawa couplings $\left(n_{g}=4\right)$ for the third and fourth families at $m_{4}=200 \mathrm{GeV}$ and $m_{L} / m_{Q}=1 / 2$. The upper and lower curves correspond to the Higgs masses, respectively, for the upper and lower Higgs critical curves shown in bold in Fig. 8. 


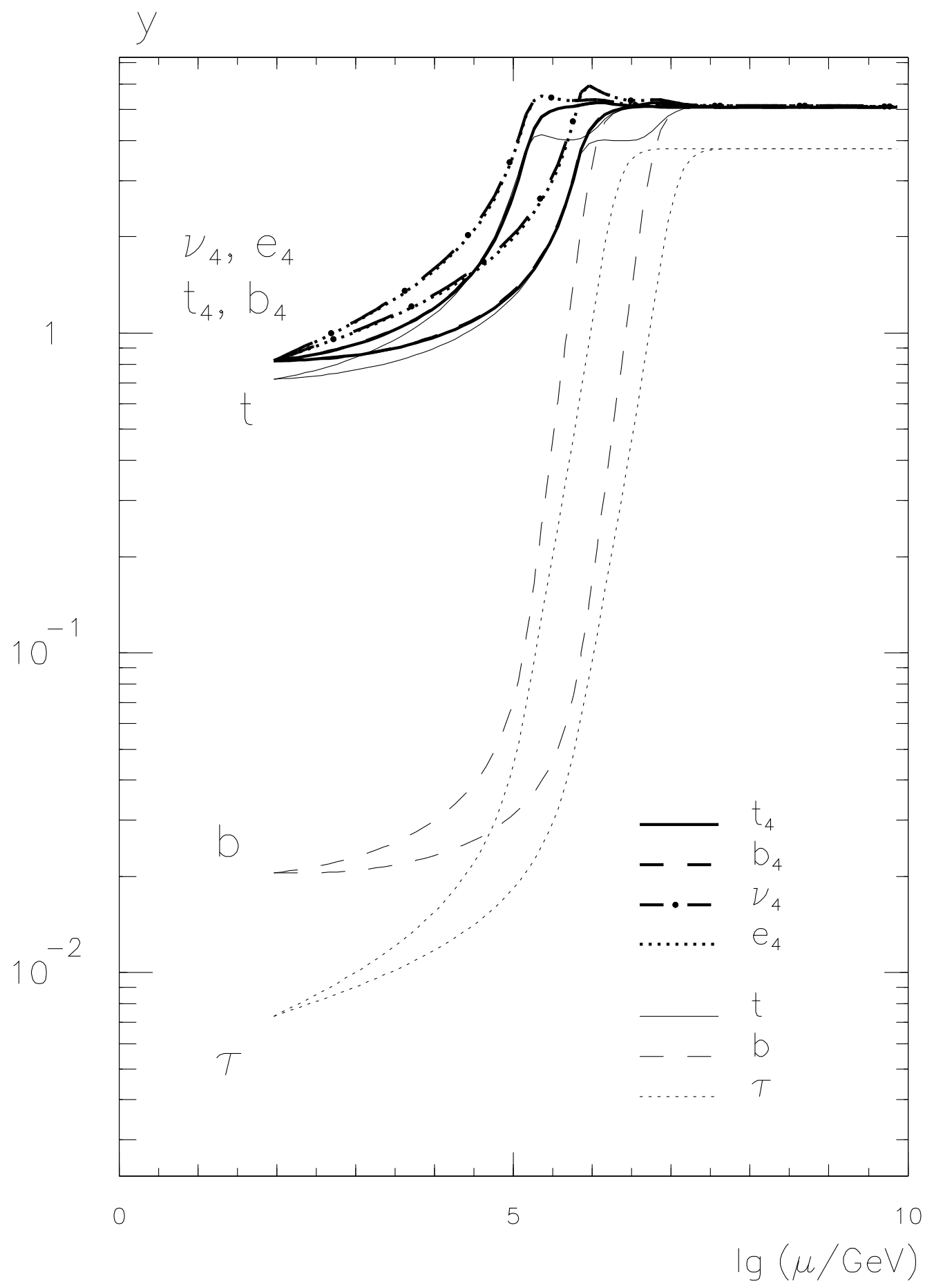

Fig. 7b: The same as in Fig. 7a at $m_{L} / m_{Q}=1$. 


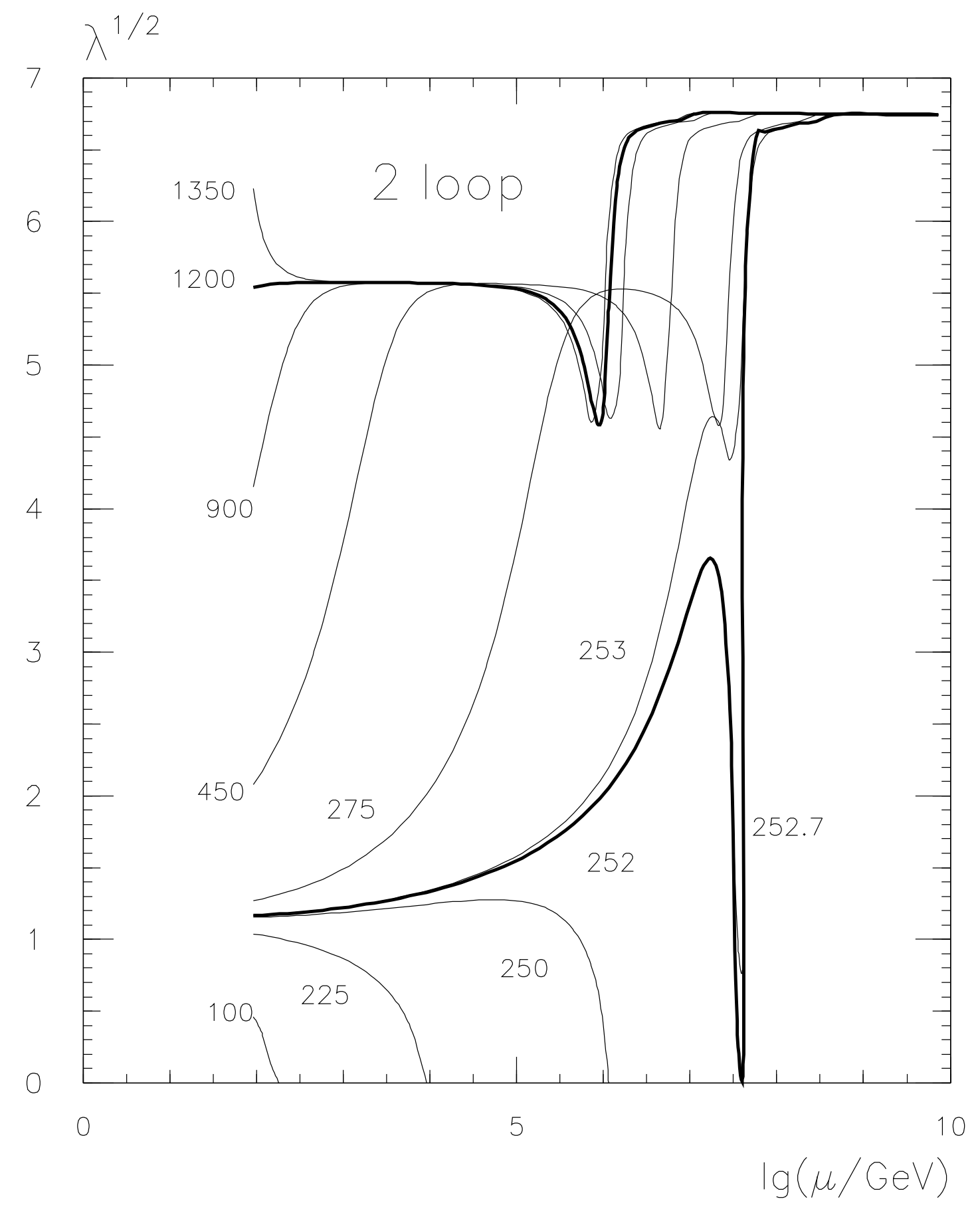

Fig. 8a: Two-loop running of the Higgs quartic coupling $\left(n_{g}=4\right)$ at $m_{4}=200 \mathrm{GeV}$ and $m_{L} / m_{Q}=1 / 2$. 


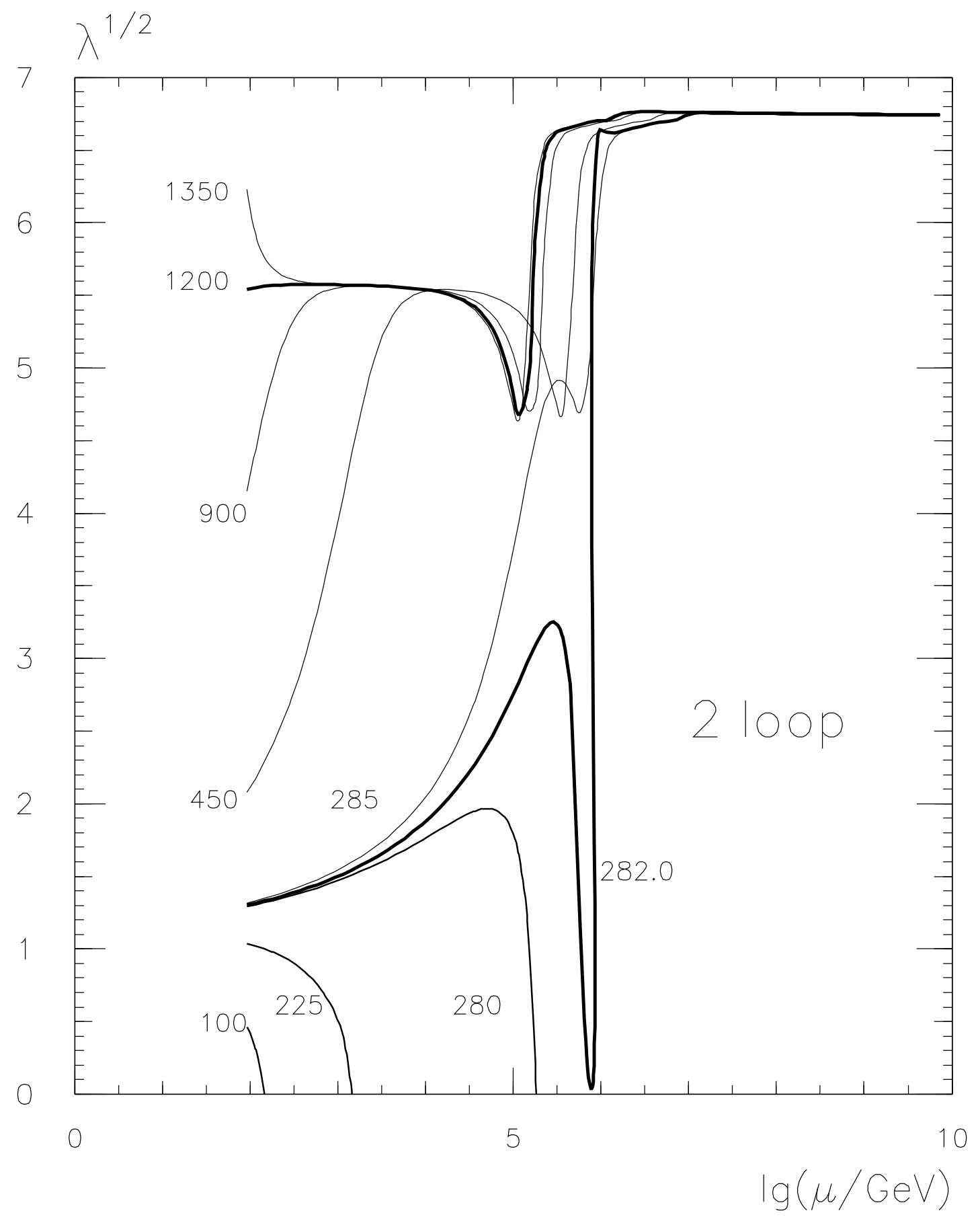

Fig. 8b: The same as in Fig. 8 a at $m_{L} / m_{Q}=1$. 


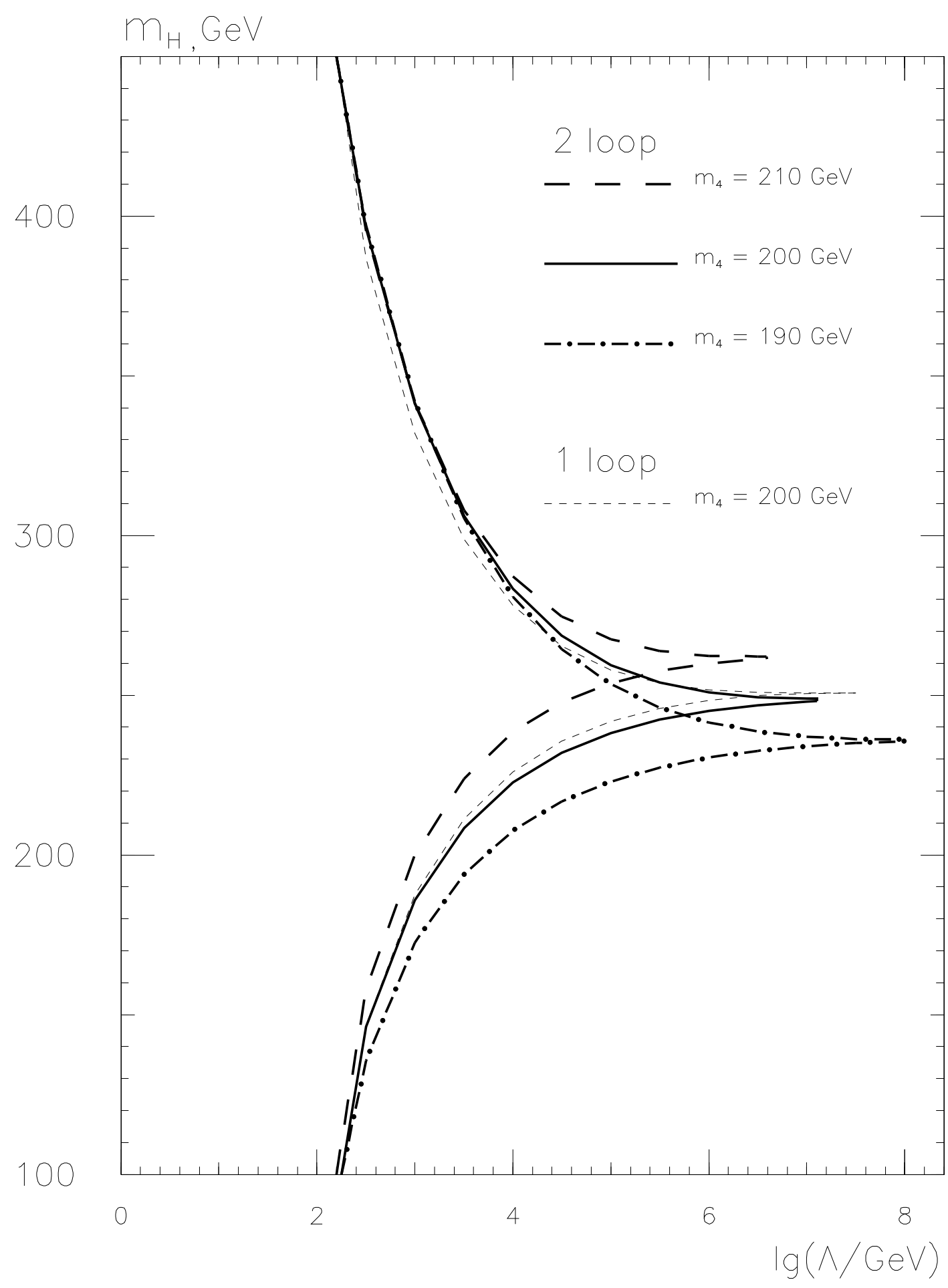

Fig. 9a: One- an two-loop self-consistency plot $\left(n_{g}=4\right)$ : the allowed Higgs mass vs. the cutoff scale $\Lambda$ at $m_{4}=200 \mathrm{GeV}$ and $m_{L} / m_{Q}=1 / 2$. 


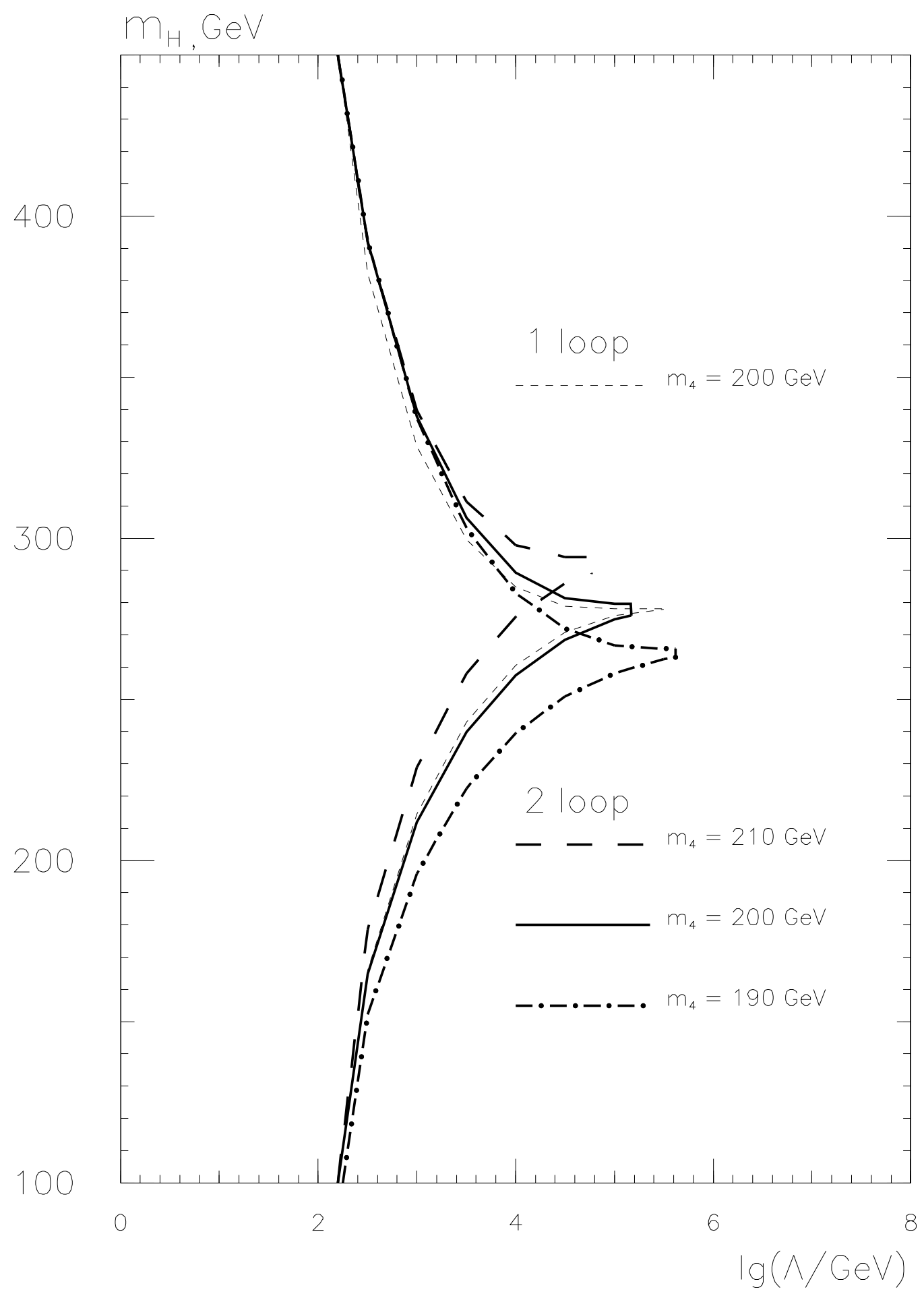

Fig. 9b: The same as in Fig. 9a at $m_{L} / m_{Q}=1$. 


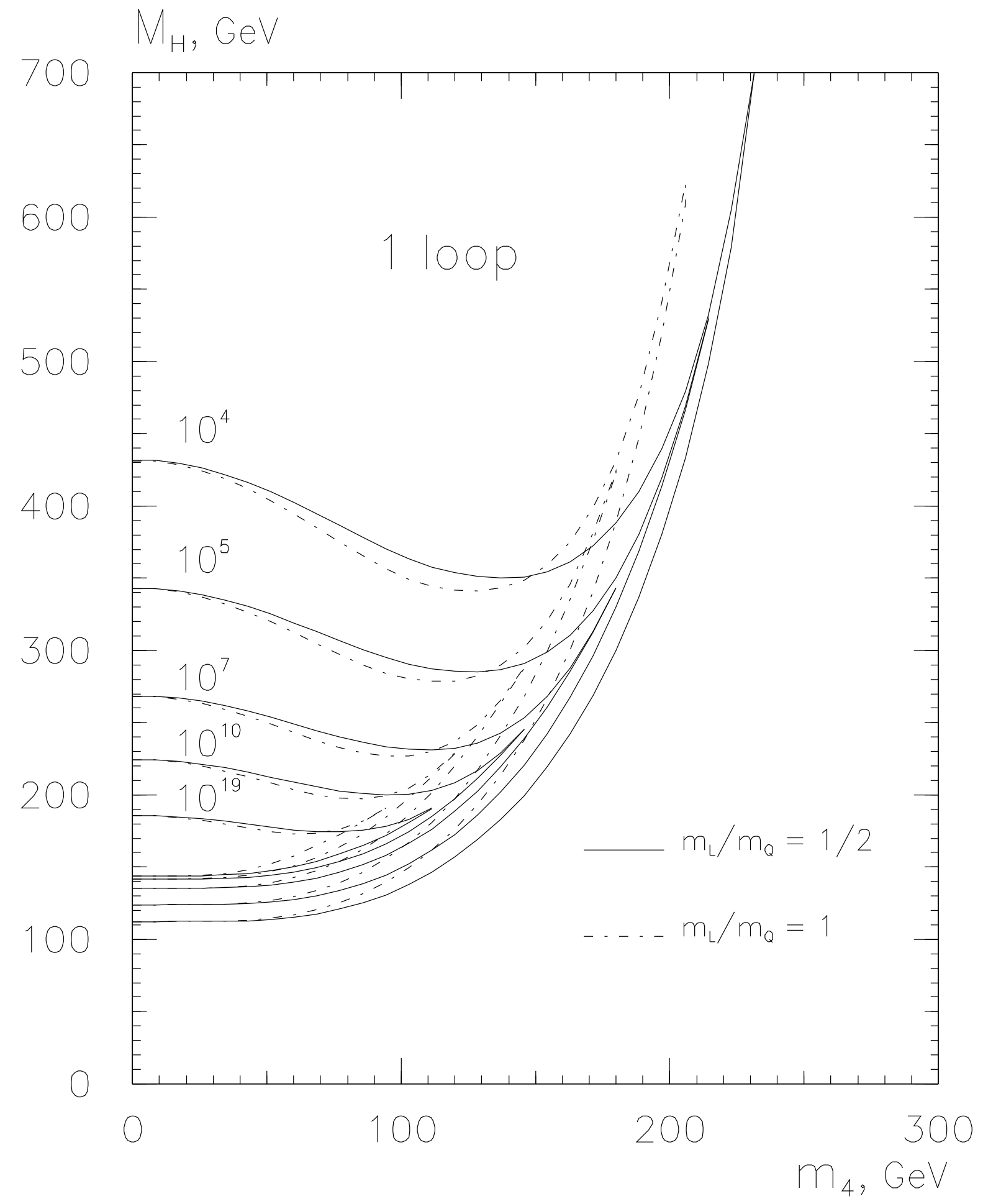

Fig. 10: One-loop self-consistency plot $\left(n_{g}=4\right)$ : the allowed Higgs mass vs. the fourth family scale $m_{4}$. The cutoff scale $\Lambda$ in GeV is fixed and $M_{t}=175 \mathrm{GeV}$. 


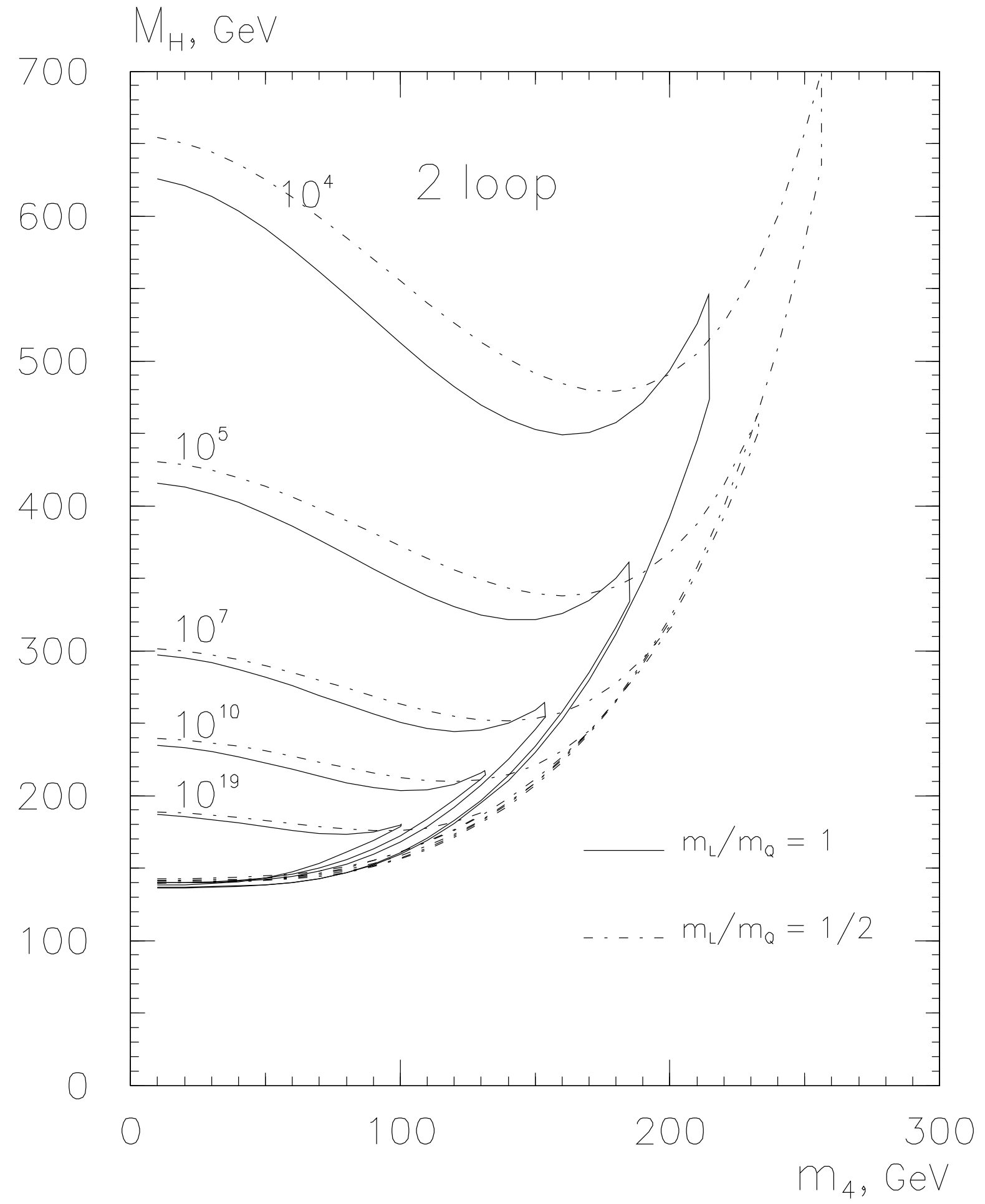

Fig. 11a: Two-loop self-consistency plot under the restriction $y \leq 1.5$ on the Yukawa couplings $\left(n_{g}=4\right)$. The rest is as in Fig. 10 . 


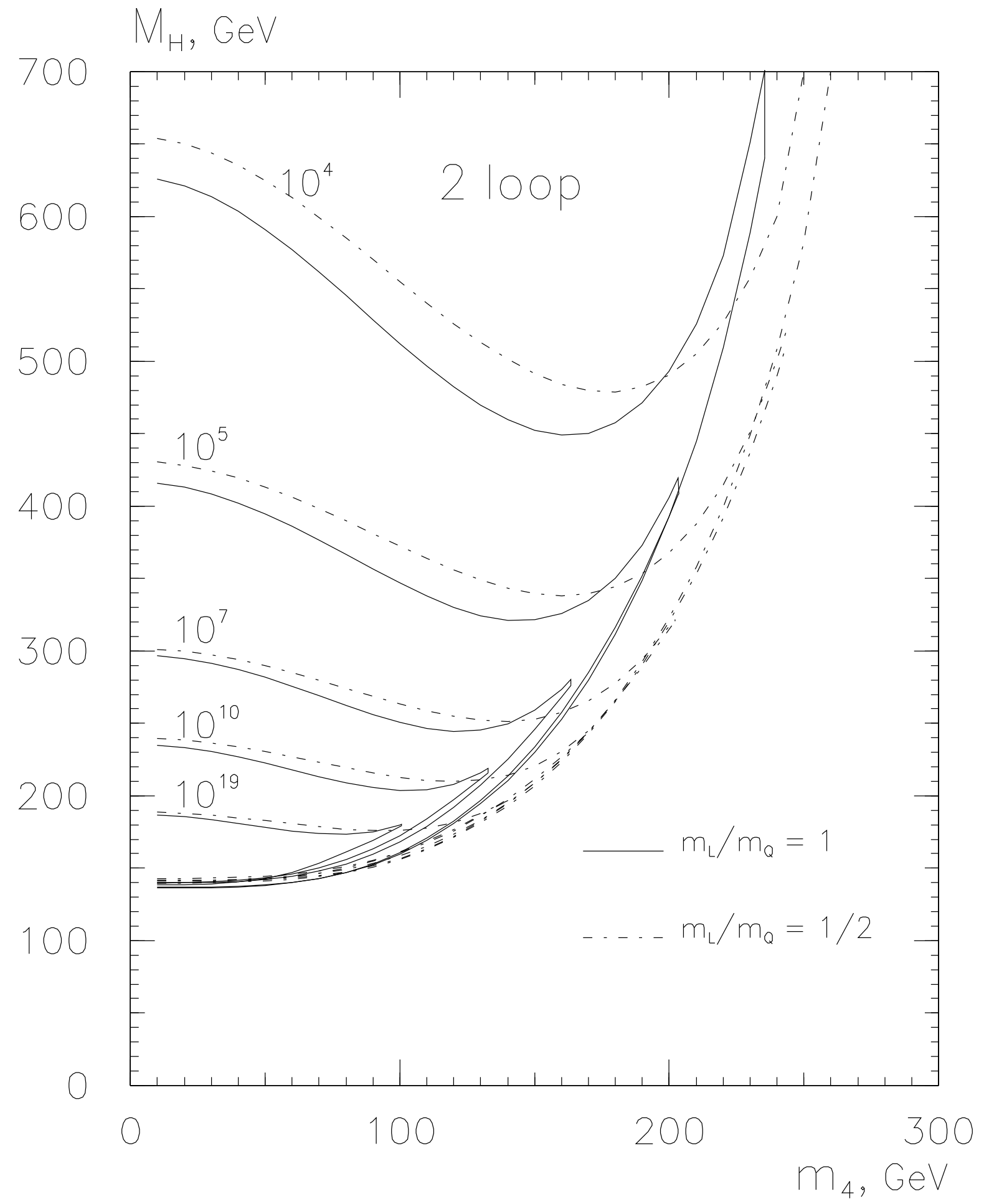

Fig. 11b: The same as in Fig. 11a at $y \leq 2$. 